Acta Crystallographica Section C

Crystal Structure

Communications

ISSN 0108-2701

Editor: George Ferguson

\title{
An $\eta^{6}$-Toluene Complex of Neodymium: $\left[\mathbf{N d}\left(\eta^{6}-\mathrm{C}_{6} \mathbf{H}_{5} \mathbf{C H}_{3}\right)\left(\mathrm{AlCl}_{4}\right)_{3}\right]$
}

\author{
Q. Liu, Y.-H. Lin and Q. Shen
}

This electronic document was scanned from an archival copy of material deposited to accompany a paper published in an IUCr journal. In many cases the only accessible copy was a microfilm of a poor-quality original. 


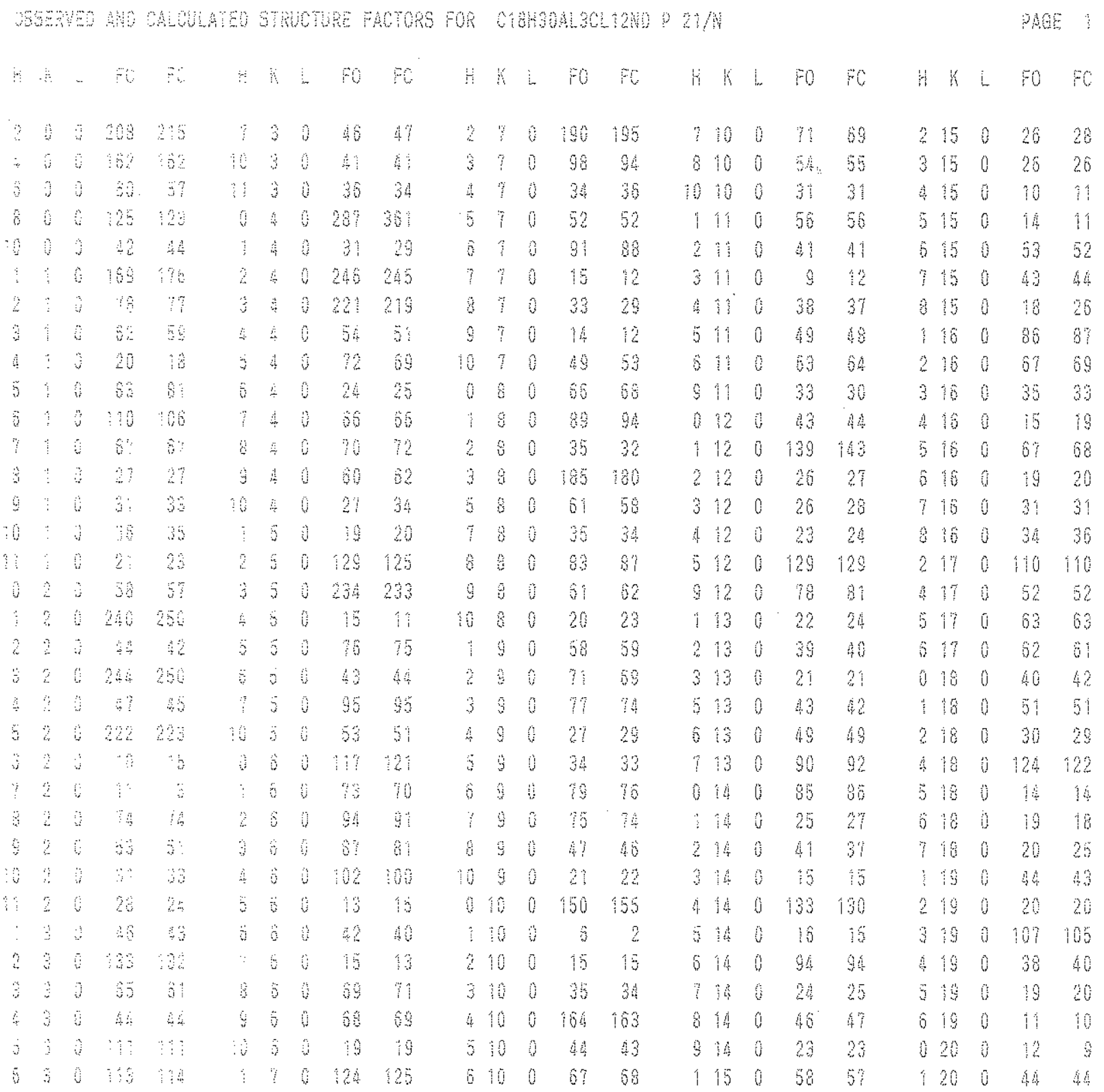




\begin{tabular}{|c|c|c|c|c|c|c|c|c|c|c|c|c|c|c|c|c|c|c|c|c|c|c|c|c|}
\hline 4 & is & 3 & $p$ & 50 & $t$ & t & - & po & $P C$ & 4 & $k$ & 1 & 10 & 80 & $\mathrm{~b}$ & 6 & $\mathrm{~L}$ & 90 & $\mathrm{Fo}$ & $\|$ & $k$ & $L$ & 50 & 80 \\
\hline 2 & 20 & 3 & $\because$ & 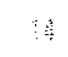 & 3 & 8 & 1 & 114 & 110 & 3 & 2 & 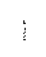 & 152 & 149 & $-\hat{4}$ & 4 & 1 & 10 年 & 100 & 8 & 5 & $i$ & 48 & 4 \\
\hline 3 & 20 & 0 & $5 \overline{5}$ & 85 & 1 & 0 & 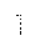 & 122 & 128 & A & 2 & 1 & 270 & $28 \%$ & -3 & 4 & 1 & 31 & 33 & 10 & 5 & & 53 & $\because 3$ \\
\hline$\therefore$ & 20 & 0 & 72 & $\because$ & 9 & 0 & 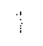 & 130 & 140 & 5 & 2 & 1 & i & 9 & -2 & 8 & 1 & 24 & 23 & -10 & $\hat{i}$ & $\hat{1}$ & 14 & \\
\hline 5 & 20 & 8 & 3 & 3 & $\cdots$ & $i$ & 1 & 38 & 4 & $\hat{0}$ & 2 & $\hat{i}$ & 23 & 27 & $-\hat{1}$ & $\hat{4}$ & 1 & 82 & 83 & -9 & 0 & $\hat{1}$ & 4 & 8 \\
\hline 3 & 80 & 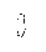 & 5 & : & -10 & 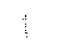 & $i$ & 54 & 80 & 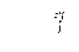 & 2 & 1 & 40 & 38 & 0 & 4 & $i$ & 10 & 16 & -8 & 6 & $\hat{\imath}$ & 32 & \\
\hline 1 & 25 & 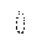 & 8 & 3 & -8 & : & 1 & 24 & 20 & 8 & 2 & $\hat{\imath}$ & 80 & 80 & $i$ & 4 & $\hat{\imath}$ & 182 & 164 & -7 & $\hat{0}$ & $\hat{1}$ & 48 & \\
\hline 3 & 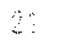 & $\hat{y}$ & 35 & 35 & - & 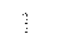 & 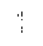 & 80 & 37 & 3 & 3 & 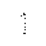 & 40 & 45 & 2 & $H$ & 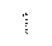 & 48 & Hi & -6 & 6 & $\hat{\imath}$ & 73 & \\
\hline 3 & 2 & 0 & 34 & 3. & $-\ddot{b}$ & 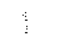 & 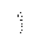 & 39 & 35 & 10 & 2 & 1 & 20 & 33 & 3 & i & 1 & 10 & 7 & -5 & 6 & 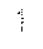 & $1 \hat{i}$ & \\
\hline 4 & $2 ?$ & , & 24 & 28 & -5 & 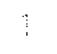 & 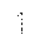 & 17 & 75 & -11 & 3 & 1 & 47 & 45 & 4 & 4 & 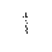 & $13 \%$ & 136 & $-i_{r}$ & 6 & 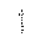 & 212 & 210 \\
\hline 5 & $2:$ & i & 18 & 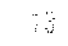 & -3 & 1 & 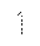 & 61 & 80 & $\cdots$ & 3 & 1 & 16 & 15 & 5 & 4 & 1 & 22 & 25 & $m$ & $\hat{a}$ & $\hat{\imath}$ & 19 & \\
\hline 8 & 3 & i & $2 z$ & 25 & -3 & i & $\vdots$ & 34 & 32 & $-\beta$ & 3 & $\hat{1}$ & 15 & 16 & $\hat{0}$ & it & $i$ & 31 & 36 & -2 & 0 & 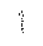 & 104 & 142 \\
\hline 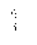 & 22 & : & $9:$ & 8 & -2 & $\because$ & 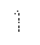 & 165 & 170 & -1 & 3 & 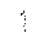 & 9 & 8 & s & 4 & 1 & 11 & 76 & $\ldots$ & 6 & $\hat{\imath}$ & 68 & 68 \\
\hline 2 & 22 & i & 2 & 16 & $\sim \vdots$ & 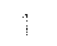 & $\vdots$ & 26 & 125 & -6 & 3 & 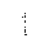 & 112 & 112 & 8 & 4 & $\ddot{i}$ & 40 & 41 & 0 & $\hat{3}$ & 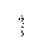 & 32 & 2 \\
\hline$\hat{3}$ & 28 & : & 28 & 8 & 0 & $\vdots$ & $\overline{1}$ & 23 & 22 & -5 & 3 & 1 & $1 \%$ & 172 & 0 & 4 & 1 & 53 & 52 & 1 & $\hat{0}$ & $i$ & 110 & 120 \\
\hline$\dot{a}$ & 28 & 3 & 29 & 8 & $\vdots$ & 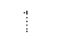 & 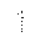 & 0 & 102 & -4 & 3 & 1 & $2 \hat{1}$ & 19 & 10 & $a$ & 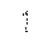 & 12 & $\hat{n}$ & 2 & 6 & 1 & 8 & \\
\hline 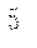 & 22 & 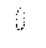 & 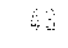 & 1 & 2 & $\because$ & 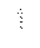 & 93 & 9 & -3 & 3 & 1 & 220 & 226 & -11 & 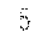 & 1 & 18 & $\hat{1} 0$ & 3 & 6 & 1 & 68 & 59 \\
\hline$\vdots$ & 23 & 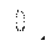 & 3 & 3 & 3 & 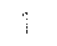 & $\vdots$ & 85 & ? & -2 & 3 & $i$ & 10 & 9 & -70 & 5 & $\vdots$ & 89 & 68 & 4 & a & 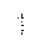 & 13 & 13 \\
\hline 2 & 28 & 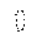 & is & ts & 5 & $\uparrow$ & $\vdots$ & 68 & 84 & -1 & 3 & 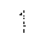 & 125 & 120 & -9 & 5 & 1 & 45 & 43 & 5 & (. & 1 & 68 & 64 \\
\hline 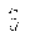 & 23 & 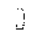 & 3 & 3 & 3 & 1 & 1 & 118 & 110 & 0 & 3 & 1 & 5 & 3 & -3 & 3 & $i$ & 3 & 31 & $?$ & 0 & 1 & 88 & 68 \\
\hline$\ddot{\Delta}$ & 23 & 6 & 20 & 28 & 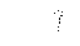 & 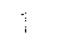 & 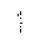 & 16 & 30 & 1 & 3 & $\vdots$ & $17 h$ & 173 & -7 & 5 & $i$ & 45 & 44 & 8 & 8 & 1 & 31 & 28 \\
\hline 3 & $B$ & 3 & 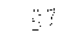 & 22 & 8 & 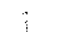 & $\because$ & 35 & $3 A$ & 2 & 3 & $\because$ & 6 & bh & $-\tilde{n}$ & 5 & $i$ & 152 & $83 \%$ & 9 & 6 & $\hat{i}$ & 19 & 1 \\
\hline 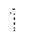 & 26 & 8 & 4 & 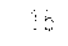 & 9 & $\vdots$ & $:$ & 5 & 40 & 3 & 3 & $\hat{i}$ & 254 & 258 & $-\ddot{b}$ & 5 & 1 & 40 & 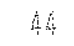 & 0 & 6 & 1 & 28 & 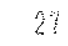 \\
\hline 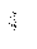 & 24 & 1 & 5 & 34 & 10 & $i$ & 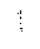 & 19 & 10 & 4 & 3 & ! & 35 & 36 & -4 & 5 & 1 & 48 & 45 & -9 & 7 & $\hat{1}$ & 34 & 36 \\
\hline 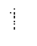 & 28 & 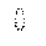 & 35 & 26 & : & ; & 1 & th & 45 & 5 & 3 & 1 & 70 & 70 & -3 & 5 & $\grave{3}$ & 53 & 54 & -7 & 7 & $i$ & 113 & 10 \\
\hline 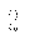 & 25 & 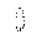 & $\because$ & 38 & $\cdots$ & 2 & $:$ & 1 & 12 & 6 & $\overrightarrow{3}$ & 1 & 121 & 121 & -2 & 5 & 1 & 302 & 393 & $\cdots$ & $\bar{y}$ & 1 & 18 & 48 \\
\hline 0 & 28 & 0 & 15 & 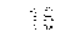 & .4 & 2 & 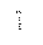 & 0 & 64 & 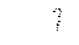 & 3 & 1 & 110 & 111 & $\cdots$ & 5 & 1 & 81 & 81 & $\cdots 5$ & 7 & 1 & 16 & 76 \\
\hline & 36 & 0 & 19 & 45 & -6 & 2 & $:$ & 152 & 131 & 10 & 3 & 1 & 39 & 40 & 0 & 5 & 1 & 11 & 11 & -4 & 7 & 1 & 62 & 58 \\
\hline$\cdots$ & 0 & 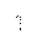 & $7 \%$ & $\because$ & $\overline{\mathrm{s}}$ & 2 & 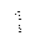 & 54 & 52 & $\cdots$ & $\overrightarrow{4}$ & i & 26 & 28 & 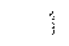 & 5 & $\hat{1}$ & 45 & 45 & -3 & $?$ & $\hat{1}$ & $1 / 4$ & 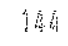 \\
\hline 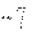 & 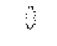 & $\vdots$ & 4 & 3 & $\cdots$ & 2 & 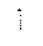 & 181 & 188 & -10 & 4 & 1 & 33 & 32 & 2 & 5 & 1 & 310 & 317 & -2 & $?$ & 1 & 37 & 35 \\
\hline-5 & 0 & $\because$ & 72 & 7 & -3 & 8 & $i$ & 30 & 85 & -6 & $i$ & 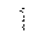 & 31 & 32 & 3 & 5 & $\hat{i}$ & 10 & 18 & -1 & 7 & 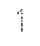 & 222 & 220 \\
\hline$\cdots$ & $\theta$ & $\vdots$ & .86 & 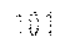 & -2 & 2 & : & 19 & TH & -3 & 4 & 1 & 47 & 47 & 4 & 5 & 1 & 97 & 96 & 0 & 7 & 1 & 3 & 10 \\
\hline-1 & 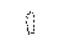 & 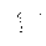 & 130 & : & 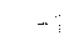 & 2 & 1 & 109 & 107 & $\cdots$ & 4 & $\hat{\imath}$ & 67 & 65 & 5 & 5 & $\uparrow$ & 13 & 13 & 1 & i & 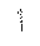 & 21\% & 226 \\
\hline$\vdots$ & i & $\vdots$ & 29 & 31 & 0 & 2 & $\hat{\imath}$ & 151 & $18 \%$ & $-b$ & 4 & 1 & 17 & 13 & $\hat{3}$ & 5 & 1 & 147 & 148 & ? & 7 & 1 & 34 & 31 \\
\hline 8 & 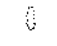 & $\vdots$ & 12 & 11" & 2 & 2 & $\vdots$ & 22 & 8 & $\cdots$ & 4 & 1 & 90 & 90 & 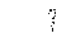 & 5 & $\hat{1}$ & 13 & 14 & 3 & 7 & $\hat{\imath}$ & 210 & 29 \\
\hline
\end{tabular}




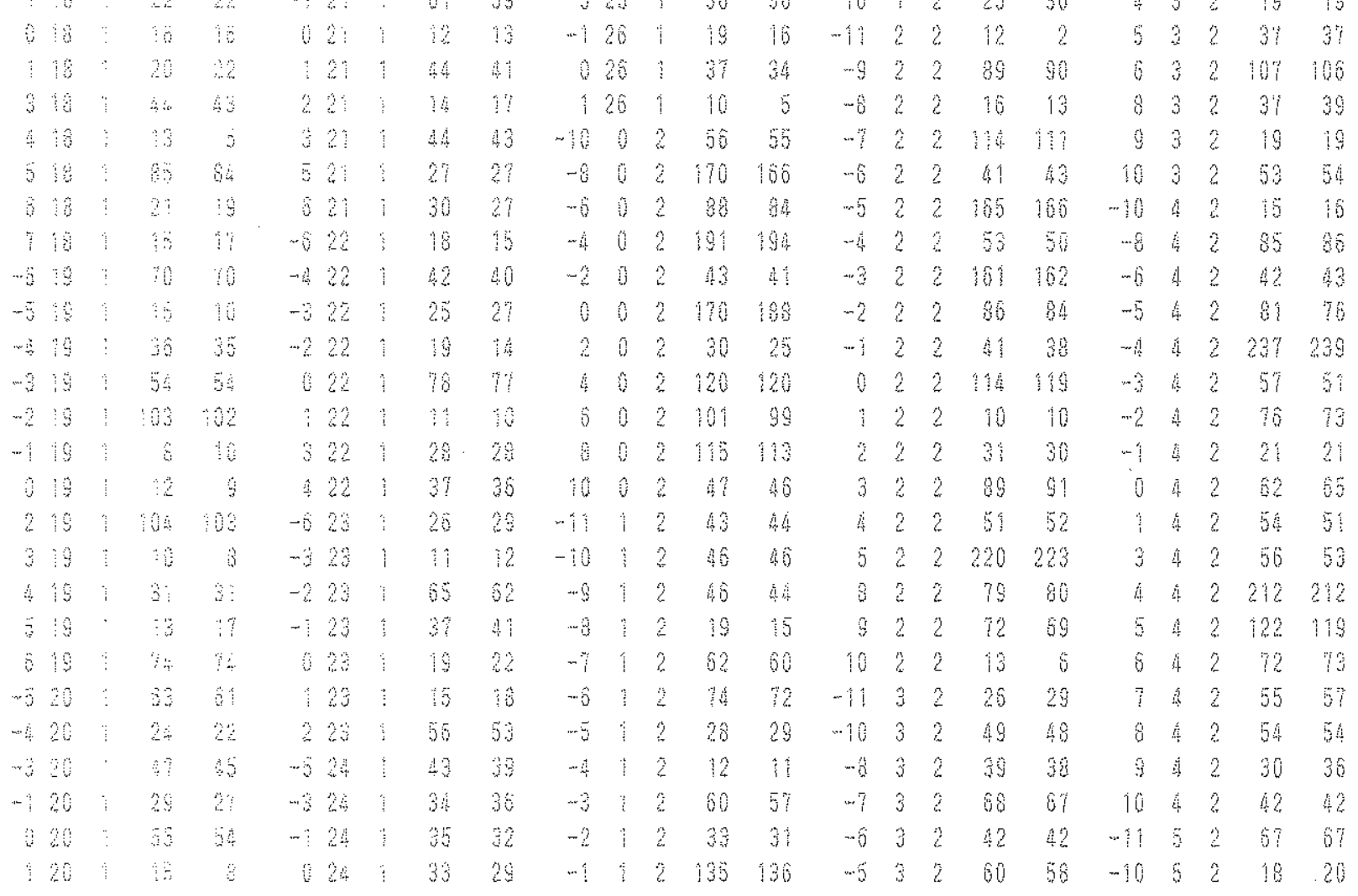

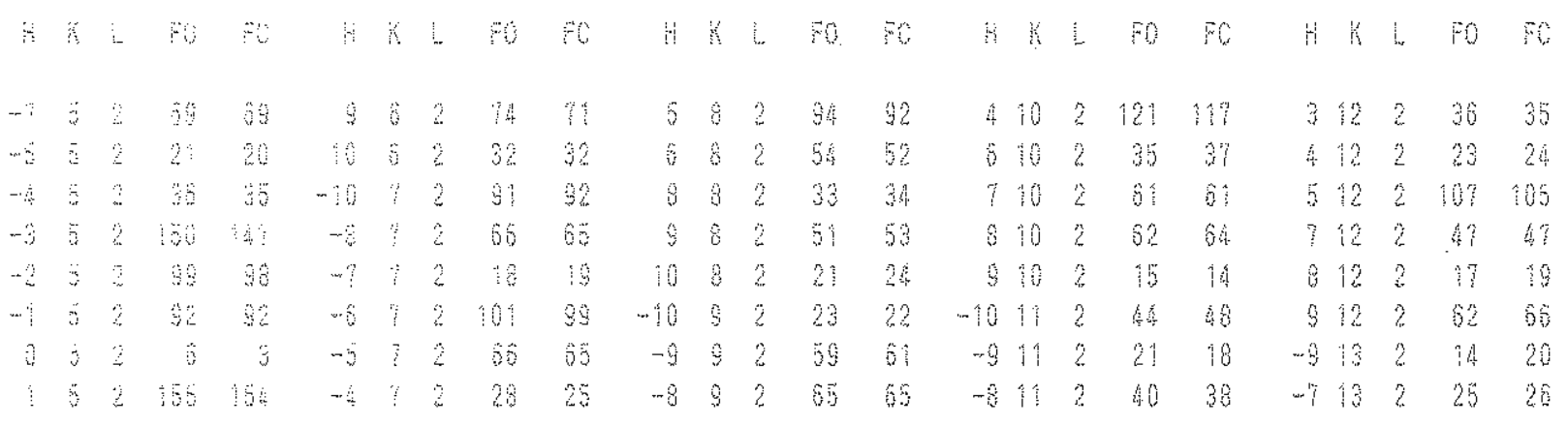




\begin{tabular}{|c|c|c|c|c|c|c|c|c|c|c|c|c|c|c|c|c|c|c|c|c|c|c|}
\hline 5 & 23 & 8 & 8 & $?$ & 2 & 96 & 90 & 1 & 4 & 2 & 103 & $10 / 4$ & 0 & 11 & 2 & 55 & 50 & 1 & 13 & ? & 50 & \\
\hline 0 & ? & 21 & $?$ & $?$ & 2 & 13 & 13 & 2 & y & 2 & 43 & 43 & 1 & $1 \%$ & 2 & 18 & 17 & 2 & 13 & 2 & 93 & \\
\hline$\hat{\theta}$ & $2 \hat{0}$ & 23 & 8 & 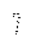 & 2 & 57 & $5 \frac{5}{5}$ & 3 & 9 & 2 & Qิ) & 7 & 2 & I & 2 & 69 & 07 & 3 & $\hat{3}$ & & 4 & \\
\hline 5 & 83 & 64 & 3 & $?$ & 2 & 13 & 13 & 4 & $\ddot{j}$ & 2 & 10 & 3 & & 11 & & 67 & 67 & & 13 & & 1 & \\
\hline 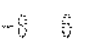 & $4 \%$ & औ & 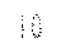 & $y$ & 2 & $5 / 4$ & 57 & 5 & 9 & 2 & 16 & 10 & & $\hat{i}$ & & 23 & 20 & & 13 & & 5 & \\
\hline 8 & $n$ & 58 & -3 & 8 & 2 & .68 & 70 & 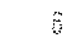 & 9 & 2 & 14 & 10 & & 11 & & 5 & 55 & 6 & 13 & & 3 & \\
\hline 6 & 40 & 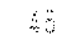 & -3 & 8 & 2 & 40 & 46 & 1 & 5 & $?$ & 47 & $4 ?$ & & 11 & & 43 & 50 & $\eta$ & 13 & & ? & \\
\hline 8 & $\because$ & 3 & -1 & 8 & 2 & 57 & 80 & 8 & 3 & 2 & 24 & 25 & & $\hat{1} \hat{\imath}$ & 2 & 40 & 37 & 8 & 13 & & $?$ & \\
\hline 6 & 180 & $16 \%$ & -6 & 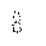 & 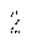 & 31 & 3 & -10 & 10 & 2 & $1 \overline{1}$ & 30 & 3 & 1 & & 15 & 18 & -9 & it & & & \\
\hline 3 & 86 & 8 & $\cdots$ & 8 & 2 & 109 & 108 & -8 & 10 & 2 & 86 & 90 & -3 & ? & & 46 & 50 & -0 & 4 & & & \\
\hline 5 & 33 & 8 & $-i$ & 6 & 2 & 11 & 170 & $-\cdots$ & 10 & 2 & 39 & 40 & $\cdots$ & 12 & 2 & 81 & 81 & $\cdots$ & 34 & & 2 & \\
\hline$\ddot{3}$ & $\mathrm{a}$ & $8 !$ & -3 & 8 & 3 & 415 & 113 & -3 & 10 & 2 & 65 & 64 & -6 & 12 & 2 & 38 & $9 ?$ & -5 & 14 & 2 & 3 & \\
\hline$\ddot{0}$ & $1 \%$ & $10 !$ & -2 & 8 & z & Aेg & 4 & -4 & 10 & 2 & 200 & 209 & -7 & 2 & 2 & 18 & 19 & $-k$ & 1 & 2 & 110 & \\
\hline 2 & " & 80 & $-\vdots$ & 8 & 2 & $8 h$ & 35 & -3 & 10 & 2 & 10 & 17 & -3 & & 2 & 126 & 12 & -2 & 4 & & & \\
\hline 3 & $\%$ & $\because !$ & b & 8 & 3 & 32 & $\mathrm{Bn}$ & $-\cdots$ & 10 & 2 & 102 & 104 & -2 & 12 & 2 & $B$ & 84 & -1 & $1 \%$ & & 62 & \\
\hline के & $3 ?$ & 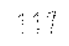 & $\vdots$ & 8 & 2 & 131 & 134 & i & 10 & 2 & 180 & 198 & $\cdots 1$ & $9 \%$ & 2 & 148 & 150 & 0 & $\mid i l$ & 2 & $18 \%$ & \\
\hline 6 & मे & $\because$ & $\%$ & i & 3 & 69 & 80 & $i$ & 10 & 3 & 29 & 31 & & 12 & 2 & 20 & 21 & & 13 & & 22 & \\
\hline 8 & $: ?$ & 2 & 3 & 8 & 2 & 29 & 29 & 2 & 10 & 2 & 119 & 120 & & & 2 & 218 & 225 & & & & $2 \hat{1}$ & \\
\hline 5 & 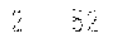 & 68 & is & 6 & 2 & 190 & $2 \mathrm{~B}$ & $\hat{3}$ & 10 & 2 & 34 & 35 & & 12 & 2 & 60 & 00 & 3 & $1 / 5$ & & $3 !$ & \\
\hline
\end{tabular}

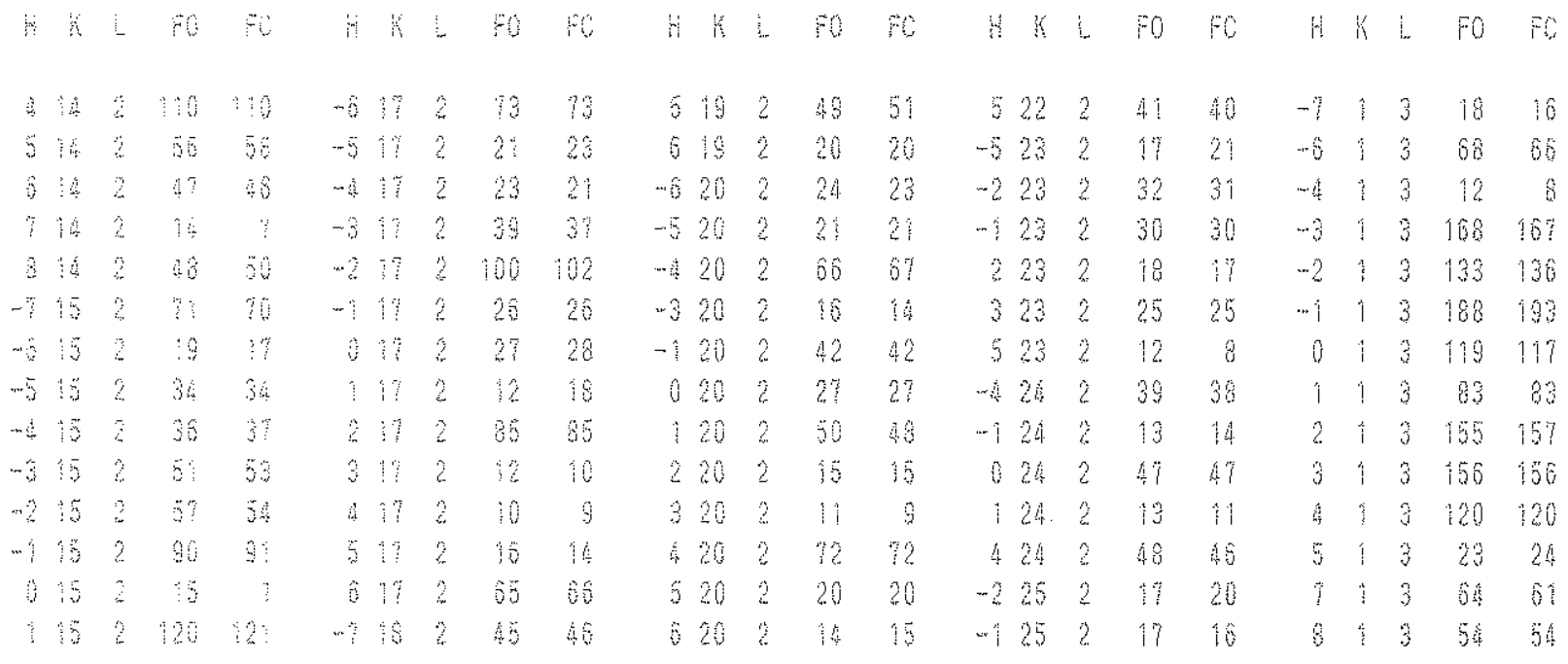




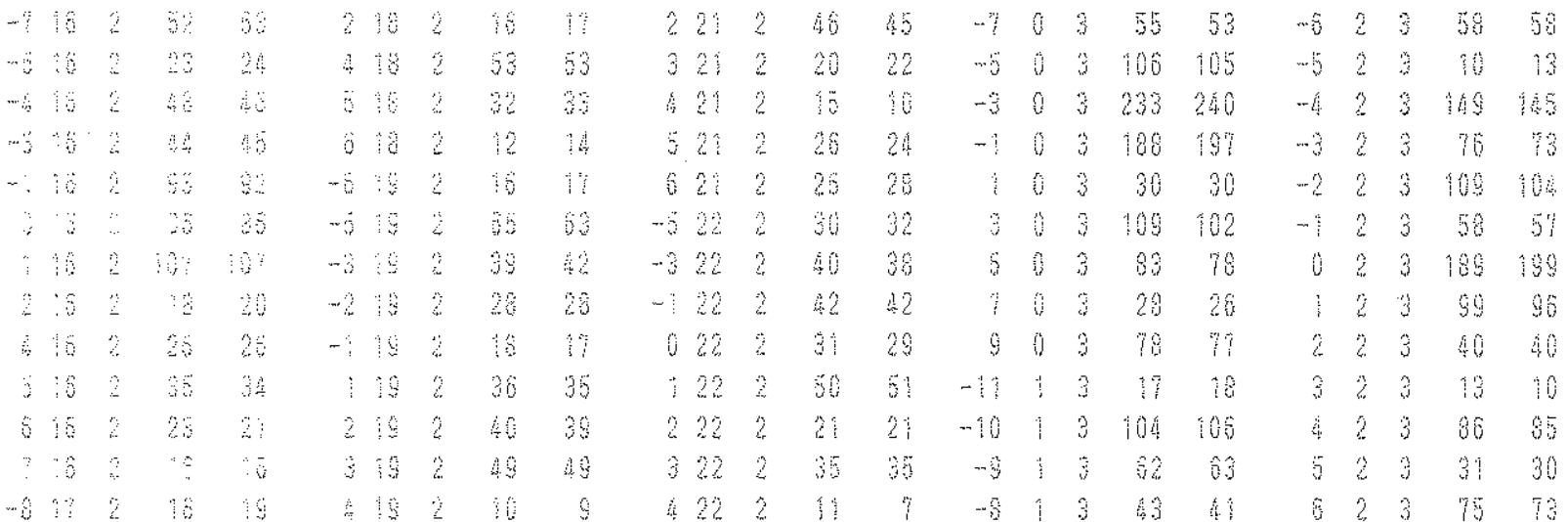

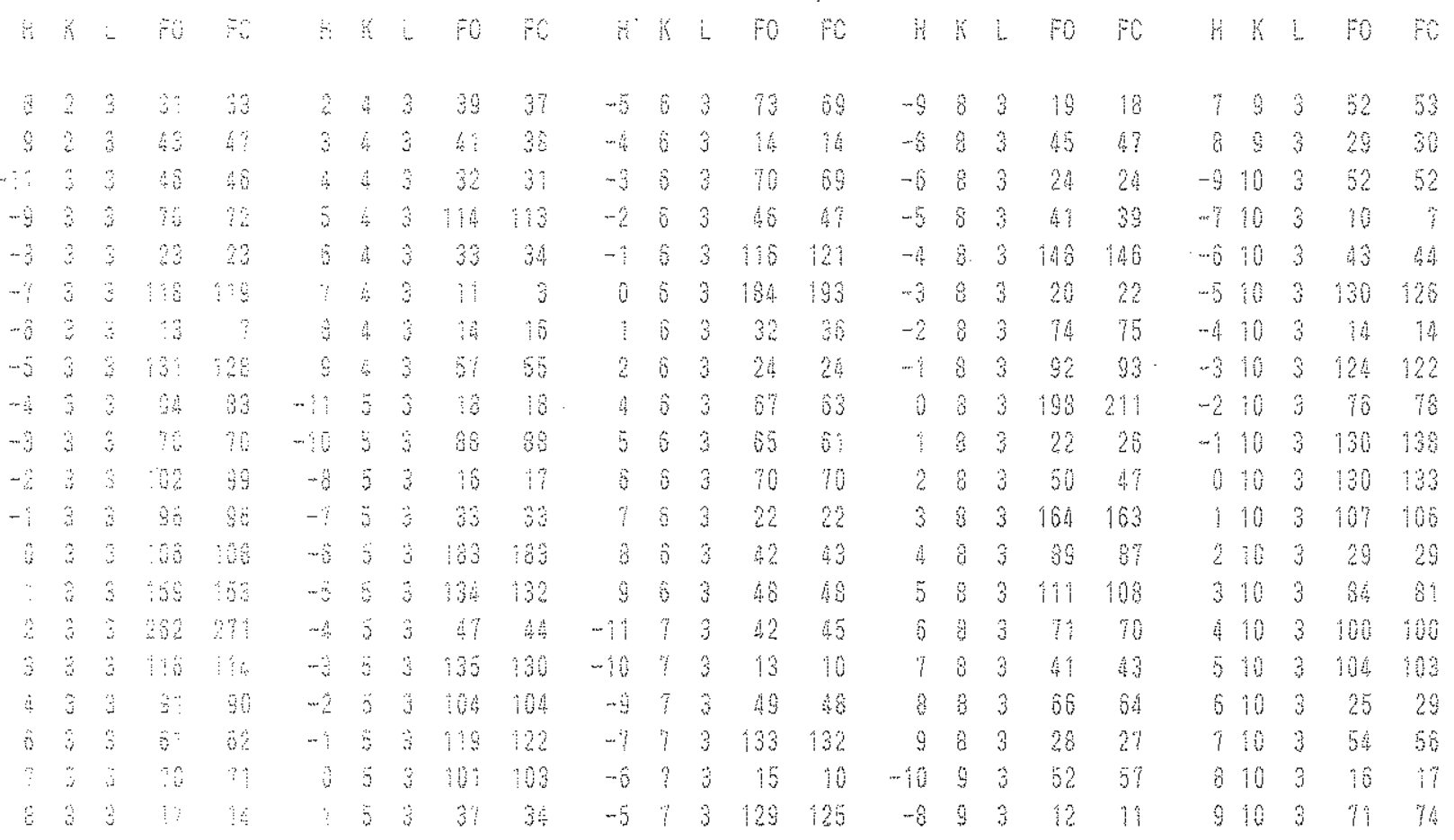




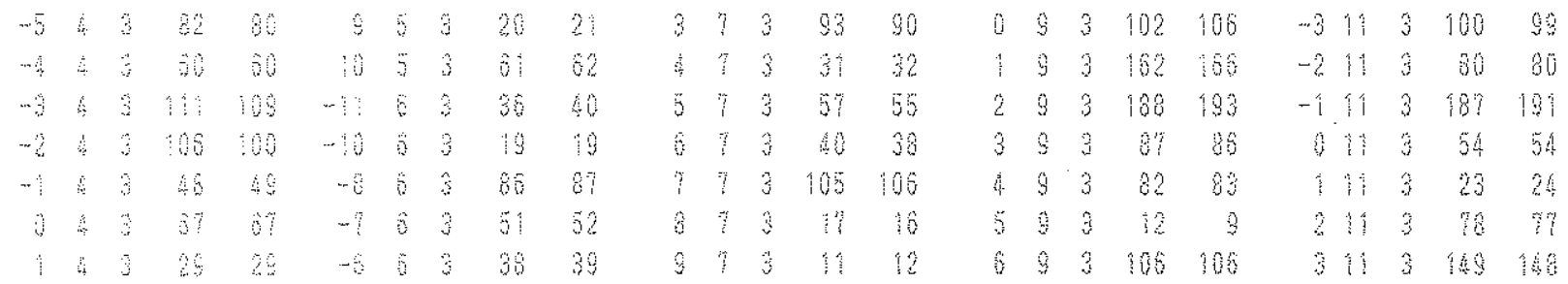

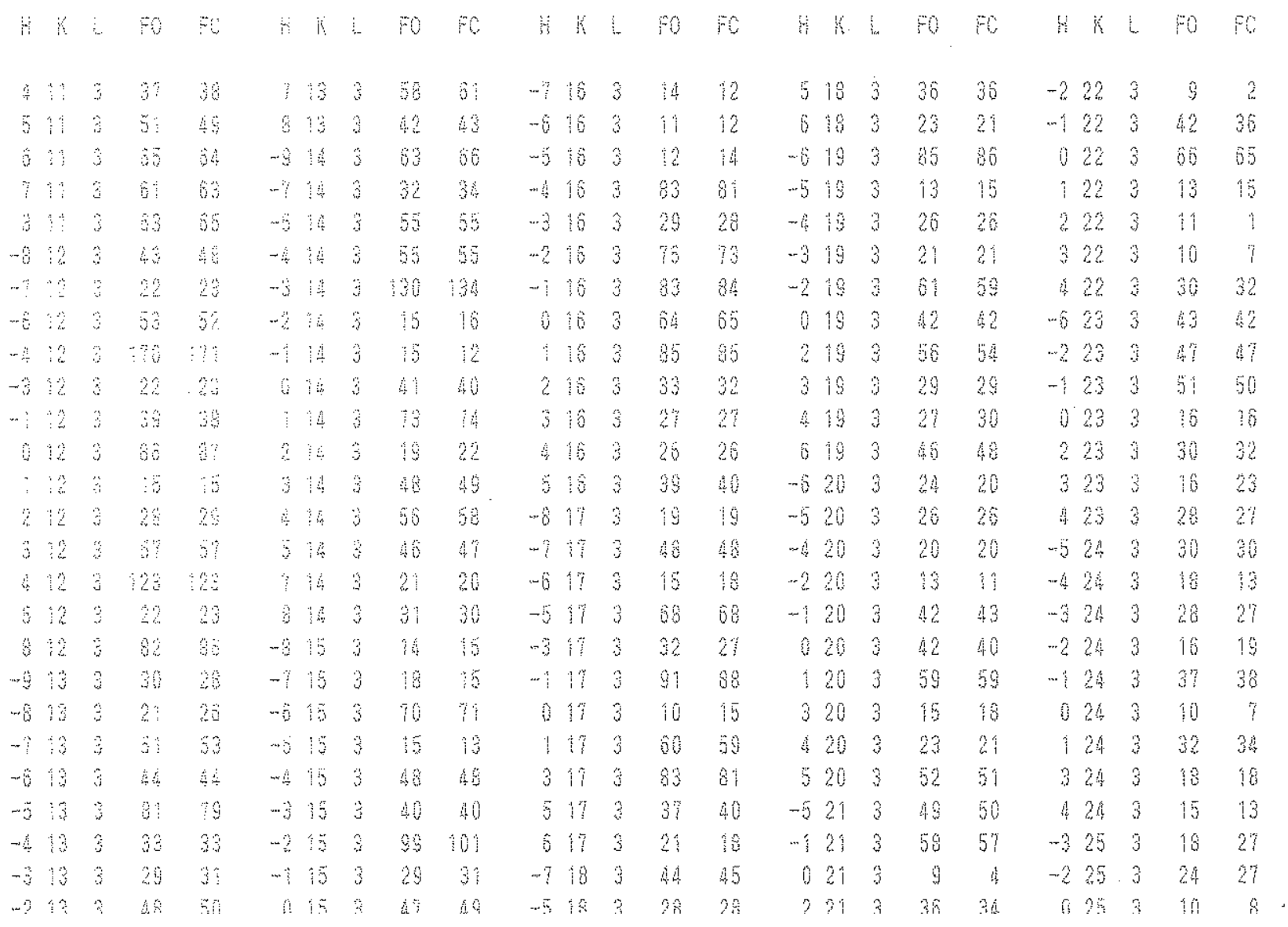




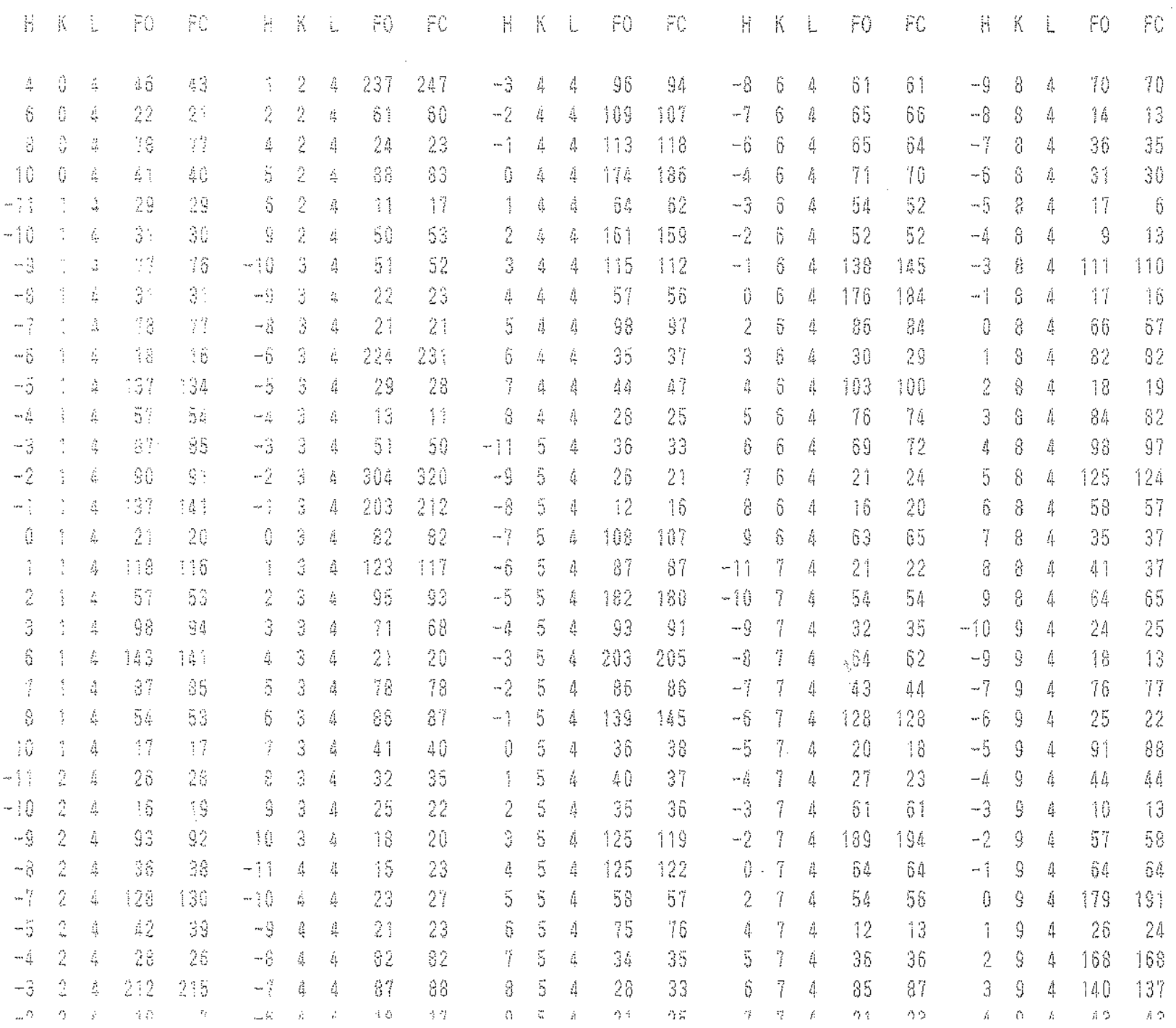




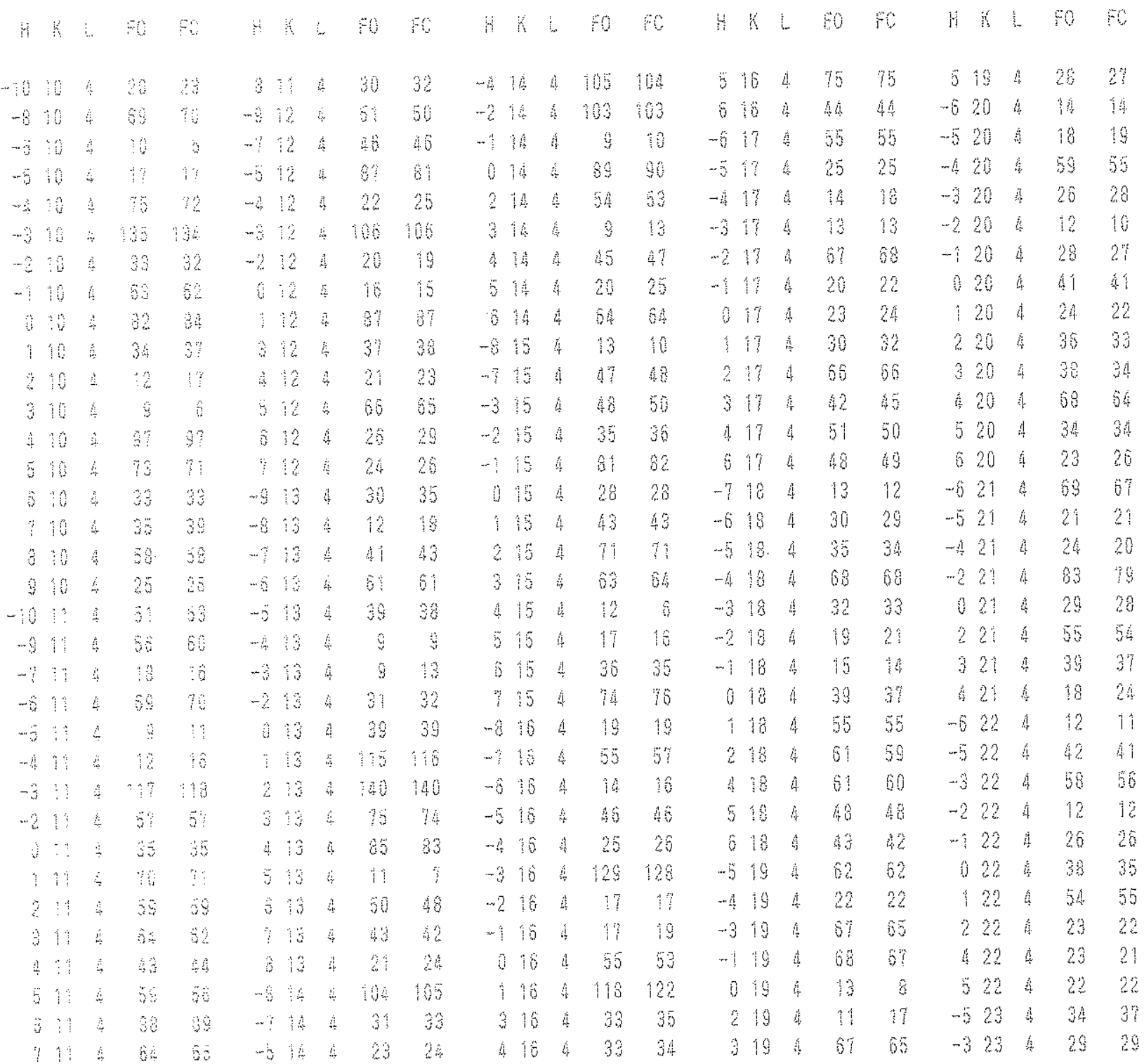




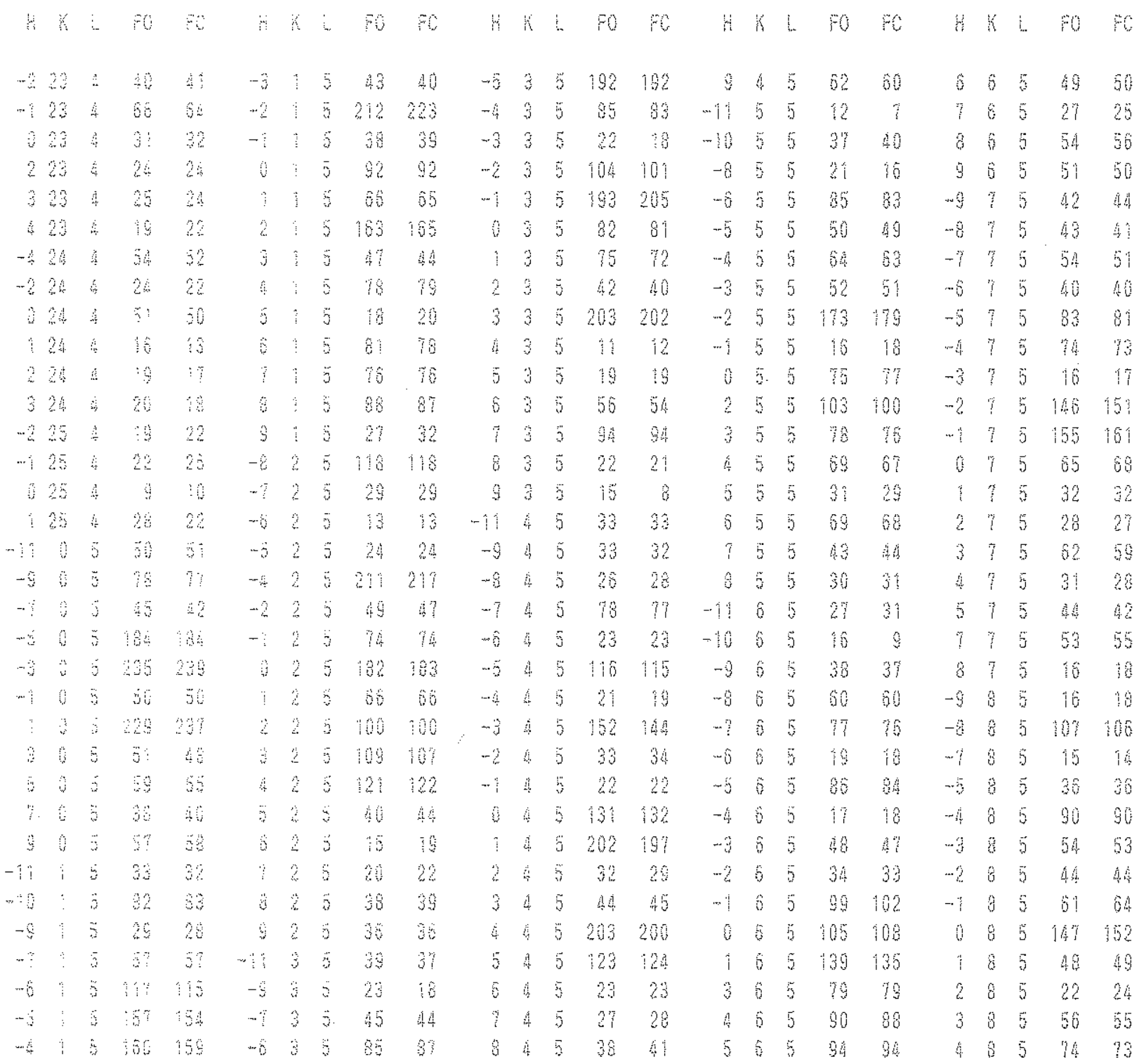




\begin{tabular}{|c|c|c|c|c|c|c|c|c|c|c|c|c|c|c|c|c|c|c|c|c|c|c|}
\hline 4 & $\vdots$ & 70 & $p$ & 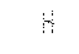 & s & - & 50 & Fo & $H$ & 6 & L & Fo & $\mathrm{Fo}$ & $H$ & K & I. & 10 & $\mathrm{PO}$ & $H$ & li & [. & 10 \\
\hline 9 & 3 & 28 & 42 & 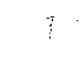 & 10 & 8 & 21 & 24 & -5 & 13 & 5 & 40 & 47 & 3 & 15 & 5 & 37 & 36 & $\cdots$ & 18 & 5 & 52 \\
\hline 8 & 5 & 14 & 4 & 8 & 10 & 5 & 18 & 20 & -4 & 13 & 5 & 42 & 43 & 4 & 15 & $\dot{5}$ & 34 & 33 & 0 & 18 & 5 & 30 \\
\hline 8 & 5 & 50 & 42 & -76 & $1 !$ & $\%$ & 25 & 3 & -3 & 13 & 3 & 43 & 44 & 8 & 15 & 5 & $\hat{1} 1$ & 9 & $\hat{\imath}$ & 18 & 5 & 60 \\
\hline 8 & 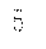 & 30 & 8 & -6 & I! & 5 & 63 & 68 & -2 & 13 & 5 & 35 & $3 ?$ & 5 & 18 & 5 & 26 & 25 & 2 & †造 & 5 & 26 \\
\hline 9 & 3 & 81 & 33 & -8 & 11 & 5 & 41 & 13 & -1 & 13 & 3 & 33 & 35 & -8 & 10 & 5 & 68 & $\hat{0} 8$ & 3 & 18 & 5 & 8 \\
\hline 0 & 5 & @ & 10 & -1 & 1 & 5 & 26 & 25 & 0 & 13 & $\sqrt{3}$ & 25 & 20 & -1 & 16 & 5 & 21 & 22 & 4 & 10 & 5 & 55 \\
\hline 3 & 3 & 3 & $\because$ & $-\hat{b}$ & 3 & 5 & 19 & 16 & 1 & 13 & 5 & 18 & 10 & -5 & 16 & 5 & 15 & 18 & 5 & 18 & 5 & 60 \\
\hline 4 & 5 & 2 & 128 & -5 & 1 & 5 & 29 & औ & 2 & 13 & 5 & 73 & 72 & -5 & 10 & 5 & 10 & $\hat{10}$ & 5 & 18 & 5 & 1 \\
\hline 3 & 5 & 28 & 36 & -3 & 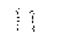 & 5 & 74 & 73 & $\overrightarrow{3}$ & 13 & 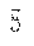 & 83 & 80 & $-\frac{\hat{b}}{3}$ & 10 & 5 & 105 & 105 & -6 & 19 & 5 & \\
\hline$G$ & 3 & 58 & 3 & -2 & $\because$ & y & 98 & 10 & 4 & 13 & 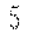 & 51 & 55 & -1 & 10 & 5 & 10 & 13 & -2 & 19 & 5 & 89 \\
\hline-3 & 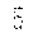 & 3 & 35 & 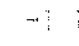 & 1 & 5 & 100 & 101 & 8 & 13 & 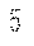 & 20 & 20 & 0 & $10 .$. & 5 & 86 & 96 & 0 & 19 & 5 & 50 \\
\hline 8 & 5 & 10 & 19 & 0 & $1:$ & 6 & 8 & 81 & i & 13 & 3 & 46 & $4 \%$ & $\hat{1}$ & 16 & 5 & 1 & & $\vdots$ & 19 & 8 & 5 \\
\hline$\because$ & $\ddot{ت}$ & 13 & 43 & $\vdots$ & 11 & 3 & 62 & 62 & $\cdots 9$ & 4 & 8 & 6 & 60 & 2 & 10 & 5 & 15 & 13 & 2 & 10 & 5 & 45 \\
\hline 9 & 5 & 4 & 50 & 2 & $" !$ & 5 & 30 & 28 & $\cdots$ & in & 5 & 41 & 40 & 3 & 16 & 5 & 32 & 32 & 4 & 19 & 5 & 50 \\
\hline 3 & 3 & 96 & 9 & 3 & 1 & 5 & 9 & 8 & -5 & 14 & 5 & 53 & 50 & 4 & 16 & 5 & 16 & 15 & $\hat{i}$ & $\hat{1}$ & 5 & 26 \\
\hline 9 & 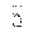 & 26 & $\hat{z}$ & $\therefore$ & $\because$ & 6 & 30 & 29 & $-i$ & $1 \mathfrak{n}$ & 3 & 18 & 8 & 5 & 10 & 5 & 27 & & $-\ddot{3}$ & 20 & 5 & 43 \\
\hline 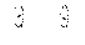 & $\ddot{3}$ & 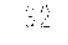 & 3 & 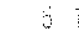 & 1 & 3 & $2 !$ & 28 & -3 & 14 & 5 & 64 & 56 & 6 & 10 & 5 & 49 & 49 & $-m / 4$ & 20 & 5 & 29 \\
\hline 9 & 5 & $9:$ & 9 & 8 & ! & 3 & $6 !$ & 52 & -2 & it & 6 & $3 \%$ & 35 & -7 & 11 & 5 & 35 & 38 & -3 & 20 & 6 & 63 \\
\hline 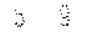 & 3 & 86 & 20 & 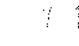 & 1 & 3 & 45 & 4 & -1 & 1 & 5 & 54 & 55 & -5 & 1 & 5 & 36 & 35 & $\cdots$ & 20 & 5 & 15 \\
\hline 9 & $\vec{b}$ & : & 10 & 8 & $\because \vdots$ & $\ddot{8}$ & 23 & 30 & 0 & 14 & 6 & 37 & 30 & $\cdots$ & î & 5 & a: & 20 & $\cdots$ & 20 & 5 & 2 \\
\hline 3 & 5 & 42 & He & -8 & 12 & 3 & 4 & 53 & 1 & It & $b$ & $11 \hat{0}$ & 119 & -3 & 11 & 5 & 70 & 11 & i & 24 & 5 & 46 \\
\hline $4 \%$ & 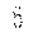 & 45 & $A$ & -6 & 12 & 3 & 20 & 10 & 2 & 14 & 5 & 31 & 31 & -7 & 11 & 5 & 10 & 17 & 1 & 20 & 5 & 83 \\
\hline 10 & $\ddot{3}$ & 80 & 33 & -6 & 12 & 5 & 14 & 15 & 4 & 14 & 5 & 38 & 29 & $\cdots$ & 11 & 3 & 72 & "1 & $\%$ & 20 & 3 & 15 \\
\hline-510 & 5 & 69 & 70 & -4 & 12 & 5 & 10 & 108 & 5 & 10 & 5 & 86 & 68 & 0 & $1 \uparrow$ & 5 & 8 & 2 & 3 & 20 & $\ddot{3}$ & 22 \\
\hline$\cdots \quad 0$ & 3 & 1 & 20 & $-\frac{G}{a}$ & $1 \%$ & 5 & 28 & $2 z$ & $\cdots$ & 15 & 5 & 16 & 11 & 2 & 11 & 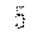 & 3 & 4 & i & 20 & 3 & 14 \\
\hline-311 & 3 & 65 & 5 & -2 & 13 & 5 & S & $?$ & -6 & 15 & 5 & 30 & 3 & 3 & १ิ & 5 & 40 & $i$ & 5 & 20 & 5 & 63 \\
\hline-210 & 3 & 8 & 10 & $\cdots$ & 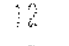 & 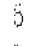 & 33 & 34 & -5 & 15 & 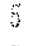 & 17 & 13 & 4 & $1 \%$ & 5 & $1 /$ & 13 & $-\hat{0}$ & 21 & 5 & 37 \\
\hline 110 & $\ddot{j}$ & 46 & 40 & 0 & 12 & 5 & 62 & 80 & -4 & 15 & 5 & 12 & 12 & 5 & î & 5 & 10 & 6 & -5 & 21 & 5 & 80 \\
\hline 30 & 5 & 2 & 3 & 2 & 12 & $\ddot{b}$ & 32 & 82 & -3 & 13 & 5 & 27 & 28 & 6 & 19 & 8 & 36 & 36 & -3 & 21 & 5 & 13 \\
\hline 10 & 5 & 08 & $0 !$ & 8 & 12 & 5 & 9 & 5 & -2 & 18 & 5 & 81 & 81 & -1 & 18 & 3 & 62 & 81 & $-\cdots$ & 2 & 5 & 106 \\
\hline 20 & $\ddot{3}$ & 10 & 15 & 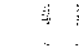 & 12 & 5 & 49 & 48 & -1 & 15 & 3 & 40 & 40 & -5 & 10 & 5 & 40 & 39 & 0 & $2 i$ & 5 & 16 \\
\hline 410 & 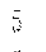 & 3 & 5 & 8 & 12 & 5 & 32 & 3 & 0 & 16 & 5 & 20 & 20 & $-\hat{n}$ & 18 & 3 & 56 & 56 & 2 & 21 & 5 & 22 \\
\hline $3: 3$ & $\ddot{3}$ & 4 & 42 & -91 & 13 & 5 & 36 & 37 & 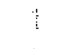 & 13 & 6 & 30 & 28 & -3 & 18 & 5 & 36 & 34 & 2 & 21 & 5 & 85 \\
\hline 610 & 5 & 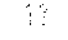 & i & -6 & 1 & 5 & 34 & 4 & 2 & 15 & 5 & 35 & 35 & -2 & 18 & 5 & 11 & $1 \mathrm{~s}$ & $\%$ & 21 & 5 & 28 \\
\hline
\end{tabular}




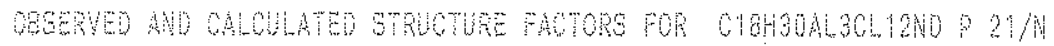

$$
\begin{aligned}
& \text { PAGE } 13
\end{aligned}
$$

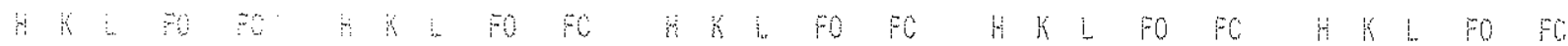

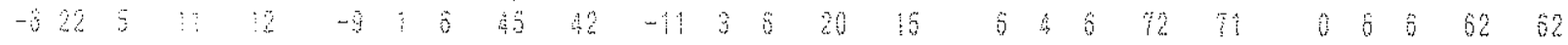

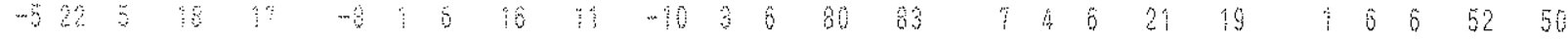

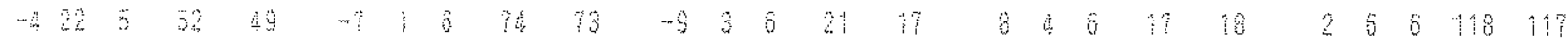

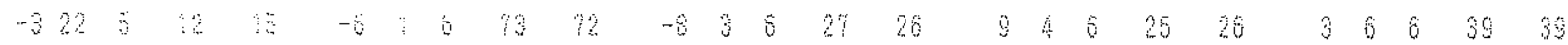

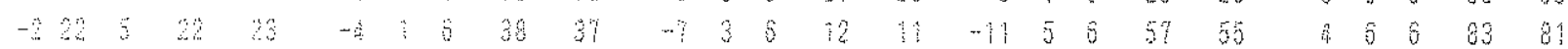

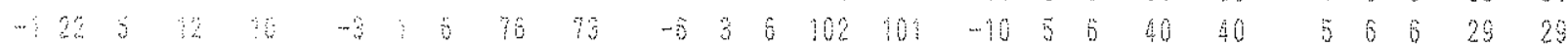

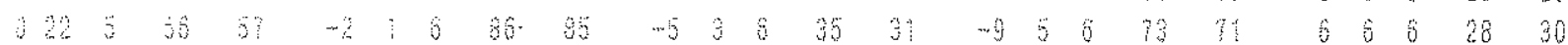

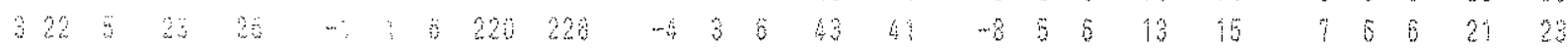

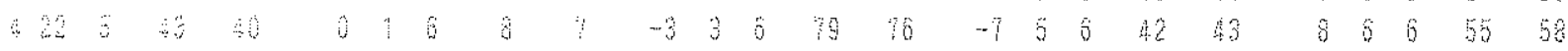

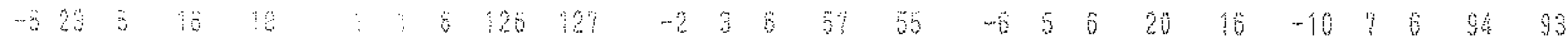

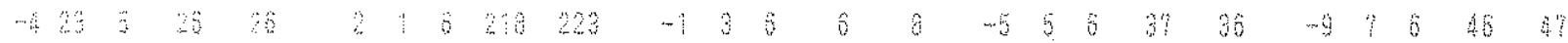

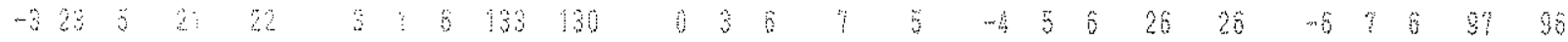

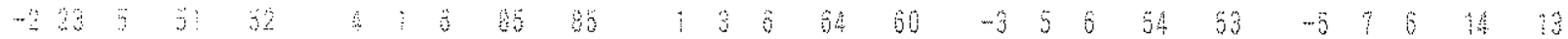

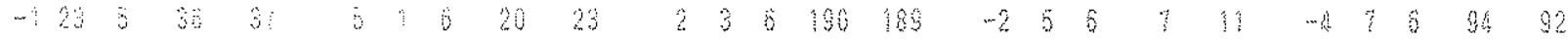

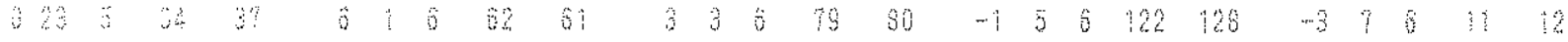

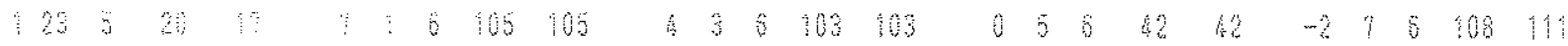

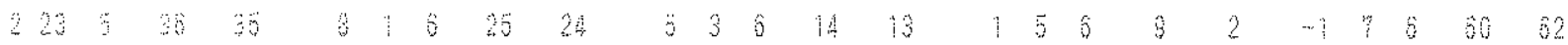

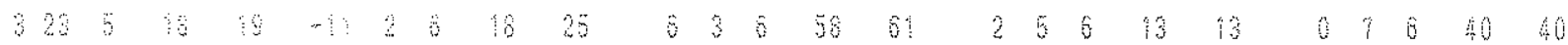

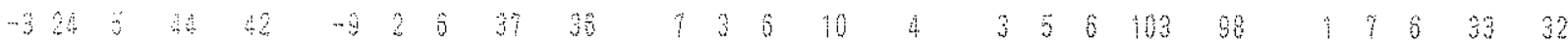

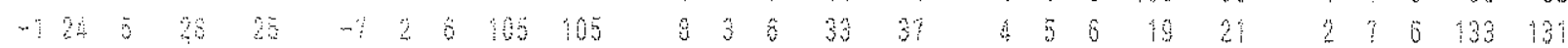

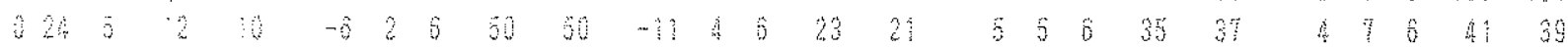

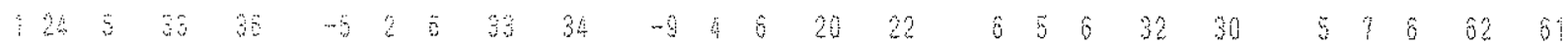

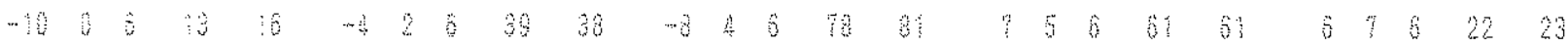

$$
\begin{aligned}
& \begin{array}{llllllllllllllllllllllll}
4 & 0 & 24 & 24 & -3 & 2 & 6 & 100 & 9 & \cdots & 4 & 6 & 35 & 32 & 8 & 5 & 6 & 14 & 12 & 7 & 7 & 5 & 31 & 31
\end{array}
\end{aligned}
$$

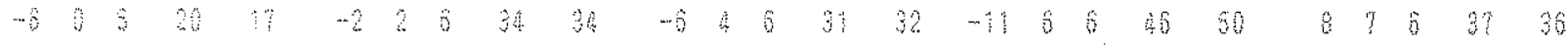

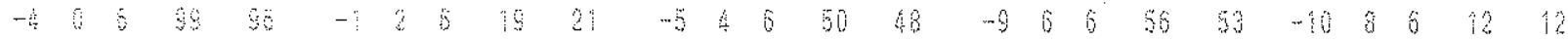

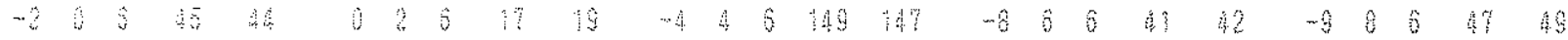

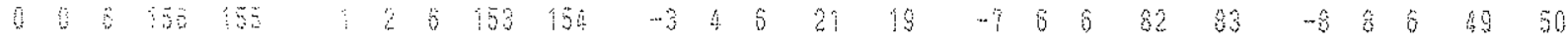

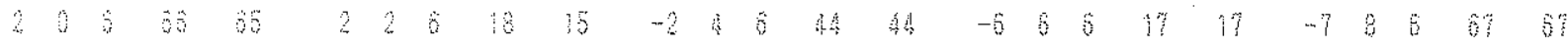

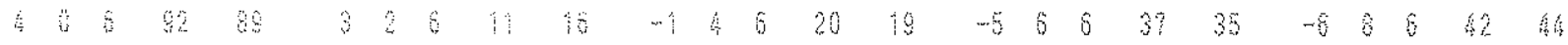

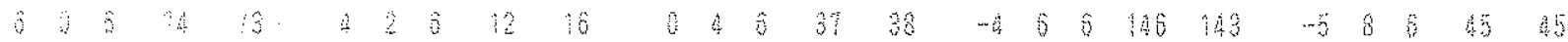

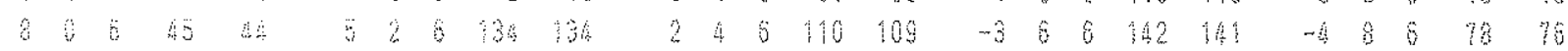

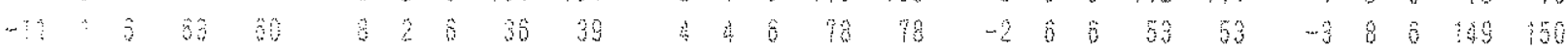

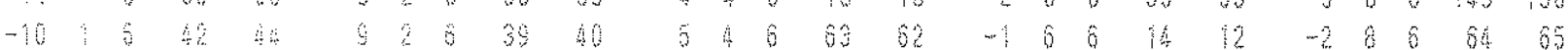




\begin{tabular}{|c|c|c|c|c|c|c|c|c|c|c|c|c|c|c|c|c|c|c|c|c|c|c|c|c|}
\hline $\mathrm{n}$ & : & $\vdots$ & 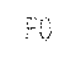 & $\mathrm{Fb}$ & 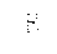 & s & 1 & $\mathrm{PO}$ & $\mathrm{C}$ & $n$ & K & 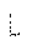 & 10 & $\mathrm{FO}$ & $\mathrm{h}$ & $k$ & $i$ & $F_{0}$ & $\mathrm{FC}$ & & 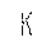 & & ro & 10 \\
\hline$-\vdots$ & $\hat{3}$ & 8 & औ & 28 & & 10 & $\hat{b}$ & 15 & 21 & 6 & 12 & 6 & 16 & 21 & $-i$ & 13 & $\hat{\mathrm{B}}$ & 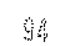 & 95 & -1 & 18 & & 46 & \\
\hline 0 & 4 & 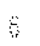 & $\because$ & 6 & 2 & 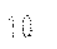 & 0 & 86 & 86 & $\cdots 9$ & 3 & 6 & 23 & 24 & 0 & 15 & $\hat{0}$ & $\eta$ & & 0 & 10 & & 3 & \\
\hline$\vdots$ & 3 & 3 & 20 & 82 & 4 & 10 & 0 & 36 & 26 & -7 & 13 & $\mathrm{~B}$ & 43 & 50 & $\bar{i}$ & 15 & 6 & 5.4 & $5 \frac{5}{5}$ & $\bar{i}$ & 18 & & १ & \\
\hline 3 & 8 & 6 & $a$ & : & 6 & 10 & 6 & 4 & 12 & $\cdots$ & 3 & 6 & 107 & 103 & 2 & 15 & 6 & 11 & 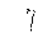 & 2 & 18 & & 6 & \\
\hline$\stackrel{?}{4}$ & 3 & 3 & : & 80 & 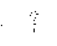 & औ & 6 & 20 & 21 & -3 & 3 & 6 & 18 & 20 & 3 & 16 & 3 & 4 & 40 & 4 & 10 & & 6 & \\
\hline$\ddot{8}$ & 8 & 6 & 8 & 85 & 8 & १॥ & 3 & 3 & 28 & 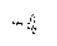 & 13 & $b$ & 12 & $1 \hat{~}$ & 4 & 15 & 6 & $\hat{\imath}$ & 13 & 5 & 19 & & 0 & \\
\hline$\ddot{3}$ & 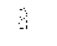 & a & 34 & 26 & -3 & 11 & 8 & 18 & 16 & -3 & 13 & 8 & 51 & 32 & 8 & 15 & 6 & $3 !$ & 39 & 6 & 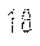 & & 17 & \\
\hline 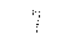 & 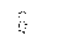 & 6 & $\%$ & & $-\cdots$ & $\because$ & $\hat{0}$ & 76 & 13 & $\cdots$ & 3 & 6 & 100 & 108 & -8 & 10 & $b$ & 30 & 30 & $\cdots 5$ & 19 & & 0 & \\
\hline-10 & 8 & 3 & 5 & 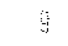 & $\cdots$ & $\because$ & 6 & 18 & 113 & -1 & 3 & 6 & 0 & 2 & $\cdots ?$ & 16 & 6 & 52 & 51 & -4 & 19 & & 8 & \\
\hline-8 & 5 & $\ddot{\partial}$ & 7 & 13 & 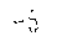 & : & 4 & 54 & 53 & 0 & 13 & $\delta$ & 68 & 10 & -8 & $1 \hat{0}$ & 8 & $2 \hat{0}$ & 25 & -3 & 19 & & 6 & \\
\hline-9 & 8 & $\ddot{z}$ & 3 & $b i$ & -3 & $\Pi$ & 6 & 30 & 39 & 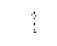 & 13 & 6 & 40 & 36 & -5 & 18 & 6 & 41 & 40 & $-\cdots$ & $\hat{\imath}$ & & 7 & \\
\hline$\cdots$ & 8 & 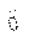 & 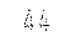 & $4:$ & -4 & 1 & 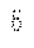 & 126 & 138 & 2 & 13 & 6 & 37 & 30 & $\ldots$ & 18 & 6 & 25 & 35 & 0 & 19 & & 5 & \\
\hline-6 & 3 & 6 & :४ & 98 & $-\vdots$ & 11 & $\hat{3}$ & 83 & 85 & 3 & 13 & 6 & 25 & 24 & $-\frac{3}{3}$ & 16 & $\hat{b}$ & 45 & $\$ 6$ & $\hat{i}$ & 19 & & $3 \hat{3}$ & \\
\hline$-i$ & 9 & 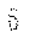 & 8 & S & 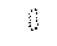 & $\because$ & 0 & $5 \%$ & 32 & 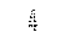 & 13 & 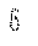 & $2:$ & 22 & -2 & 16 & 6 & 32 & 30 & 2 & 19 & & í & \\
\hline-3 & 9 & $\ddot{0}$ & 30 & 8 & $:$ & $\because$ & 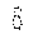 & 20 & 10 & $\hat{0}$ & 13 & 6 & 52 & 52 & $\cdots \hat{\imath}$ & 16 & 6 & 23 & 24 & 3 & 19 & & 83 & \\
\hline-2 & 9 & 8 & 48 & 5 & 2 & $\because$ & 0 & 89 & 89 & $\eta$ & 13 & $\hat{\imath}$ & 43 & 65 & 0 & 18 & 6 & $5 a$ & 53 & 6 & 19 & & 12 & \\
\hline - & $\hat{3}$ & 3 & 19 & $\$ 9$ & 3 & $\because$ & 6 & 31 & 28 & $\cdots$ & 14 & 6 & $8 \hat{i}$ & 85 & $\hat{\imath}$ & 16 & 6 & 93 & 13 & $-\tilde{i}$ & 20 & & 16 & \\
\hline a & 9 & $\hat{0}$ & 5 & 58 & $\hat{i}$ & 1 & 6 & 4 & 40 & $-n_{1}$ & 14 & $b$ & 45 & 30 & 4 & 16 & 8 & $\hat{\imath}$ & $\hat{3}$ & $-\overline{3}$ & 20 & & 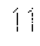 & \\
\hline$\vdots$ & 9 & 8 & $\Delta$ & th & $\ddot{3}$ & 11 & 6 & 22 & 22 & -6 & 14 & 6 & 37 & $3 \mathrm{~A}$ & 5 & $1 \hat{0}$ & $\hat{\mathrm{z}}$ & 52 & 53 & -4 & 20 & & $\hat{1}$ & \\
\hline 2 & 8 & 6 & $3:$ & 23 & 0 & $1:$ & b & 31 & 57 & -5 & 14 & 8 & 10 & 10 & 6 & 10 & $\hat{0}$ & 21 & 25 & $\cdots 3$ & 20 & 0 & 20 & \\
\hline 3 & 9 & 3 & 10 & 18 & 7 & $\|$ & $\overline{8}$ & 19 & 51 & -4 & Ha & $\hat{0}$ & 7 & 70 & -6 & 17 & 8 & 50 & 52 & $\hat{j}$ & 20 & 0 & 47 & \\
\hline 5 & 9 & 8 & is & 10 & $\cdots$ & 12 & 8 & 13 & 9 & -2 & t4 & 6 & 35 & 35 & -5 & 17 & 6 & 18 & 16 & 1 & 20 & & 26 & \\
\hline$\ddot{a}$ & 9 & $\hat{3}$ & $3:$ & 32 & -7 & 12 & $\hat{\beta}$ & 70 & $7 \overrightarrow{5}$ & $-i$ & 14 & $\tilde{3}$ & 11 & 8 & -3 & 17 & 6 & 32 & 36 & 2 & 20 & D & 31 & \\
\hline$"$ & $\mathrm{~g}$ & 8 & 50 & 6 & -6 & 13 & 6 & 16 & $1 \%$ & 0 & 3 & $\hat{0}$ & 83 & 84 & -2 & $1 ?$ & 8 & 100 & 90 & 4 & 20 & 0 & 46 & \\
\hline 3 & $\mathrm{a}$ & 8 & 85 & 28 & -5 & 12 & 6 & 57 & 80 & 2 & 14 & 0 & 25 & 23 & 0 & 17 & 8 & $3 \hat{8}$ & 38 & 5 & 20 & 8 & 18 & \\
\hline-9 & 10 & 8 & 23 & 25 & -3 & 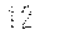 & 6 & 71 & 70 & 4 & 14 & 6 & 16 & 20 & 1 & 17 & 0 & 10 & 18 & -6 & 21 & 0 & 32 & \\
\hline$-m$ & 0 & $s$ & 3 & 86 & -2 & 12 & 6 & 19 & 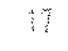 & 5 & 4 & 3 & 12 & 3 & 2 & 17 & 6 & $n$ & 48 & -5 & $2 \hat{3}$ & 6 & 20 & \\
\hline-7 & 10 & 8 & 26 & 23 & ים & 12 & 6 & 17 & $\hat{\imath}$ & 8 & $\hat{1}$ & 6 & 51 & 52 & 5 & 11 & 6 & 31 & 36 & $\cdots 3$ & 21 & 6 & 14 & \\
\hline-8 & 18 & 8 & 2 & 12 & 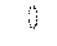 & 12 & 6 & 10 & 42 & -1 & 15 & 6 & $5 \overline{1}$ & 37 & 0 & 17 & 6 & 12 & 42 & $\cdots 2$ & 23 & 6 & 70 & \\
\hline-3 & 10 & 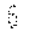 & $4 \vdots$ & 8 & 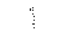 & 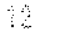 & 6 & 65 & 86 & -6 & 15 & 8 & 26 & 20 & -6 & 18 & 0 & 33 & 39 & -1 & 21 & 6 & 14 & \\
\hline 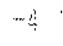 & $\because$ & 8 & $\because: 4$ & $\because ":$ & 2 & 12 & 8 & :" & 22 & -3 & 16 & 6 & 90 & 89 & -5 & 78 & 6 & 39 & $3 \%$ & $\theta$ & $2 !$ & 8 & 22 & \\
\hline-3 & 0 & 8 & 6 & $5:$ & 3 & $\because$ & 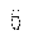 & 26 & 30 & -4 & 16 & 8 & 10 & 11 & -4 & 18 & 0 & 52 & 50 & ! & 21 & 6 & 24 & \\
\hline-2 & 0 & 8 & 30 & bे & 4 & 12 & 8 & 16 & 22 & $\cdots$ & 13 & 8 & 14 & 18 & -3 & 18 & 6 & 45 & 43 & $?$ & 21 & 0 & 50 & \\
\hline 0 & 10 & 6 & $1 ;$ & $\because$ & 3 & $1 z$ & $\ddot{b}$ & 31 & $5 \%$ & -2 & 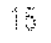 & 3 & 31 & $3 / 3$ & -2 & 18 & 6 & 15 & 斿 & 3 & 21 & & 31 & \\
\hline
\end{tabular}




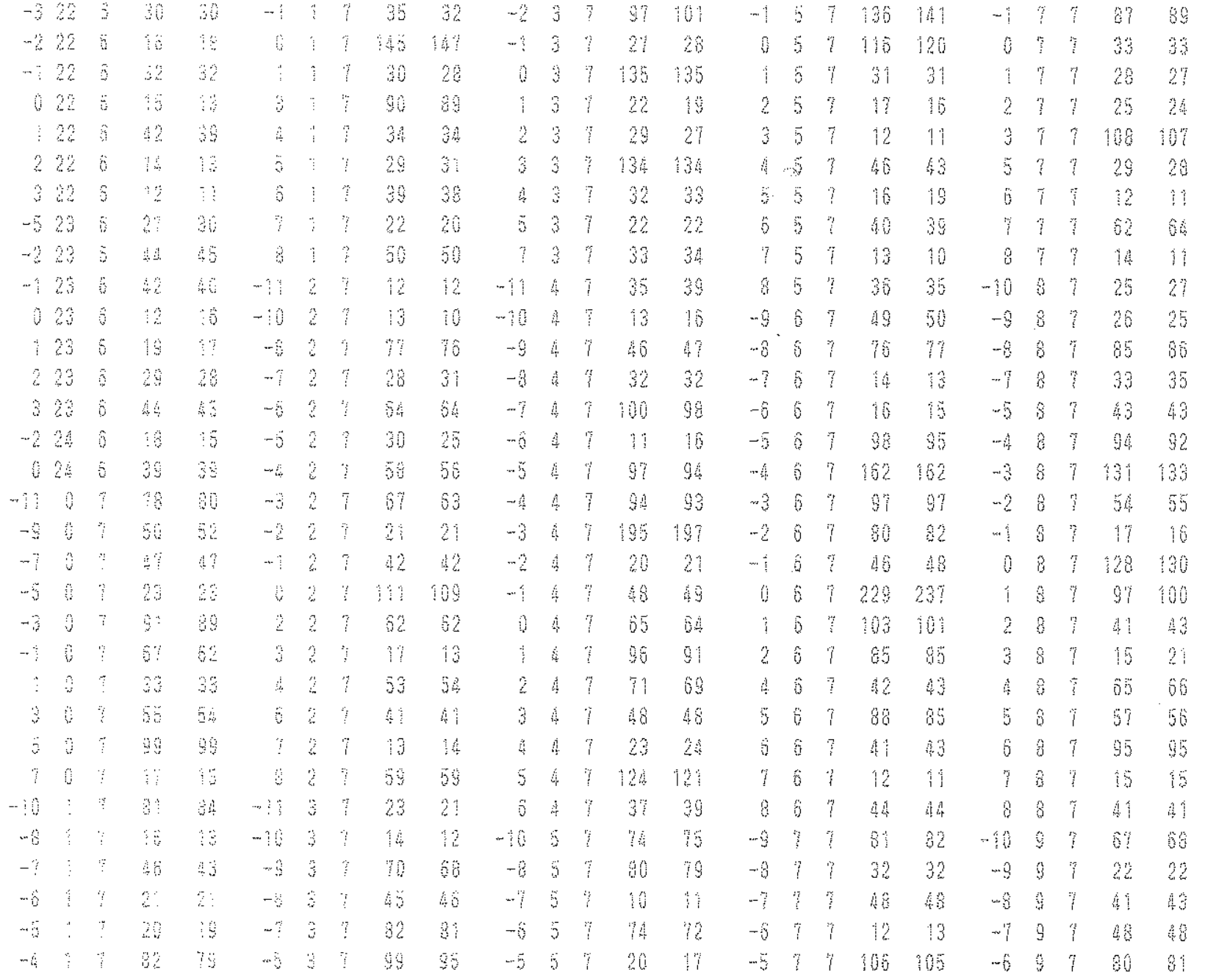




\begin{tabular}{|c|c|c|c|c|c|c|c|c|c|c|c|c|c|c|c|c|c|c|c|c|c|}
\hline 80 & 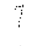 & 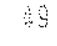 & 49 & -4 & $?$ & 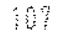 & 103 & 14 & 7 & 98 & 98 & 4 & $\hat{\mid} \mid \hat{\}}$ & 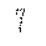 & 21 & 24 & -1 & 21 & "u & 50 & 50 \\
\hline-510 & 7 & $\bar{s}$ & 5 & $-31 \%$ & 7 & 11 & 6 & 214 & 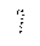 & 46 & 45 & 5 & 11 & ? & 16 & 3 & $\tilde{0}$ & $2 \hat{3}$ & 7 & 14 & 12 \\
\hline$\because \quad 0$ & 7 & 102 & 12 & -212 & 7 & 80 & 58 & 314 & 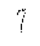 & 39 & 42 & -5 & 18 & 7 & 18 & 20 & 3 & 21 & $\overrightarrow{\mathrm{i}}$ & 16 & 10 \\
\hline-510 & 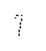 & 16 & 13 & $\begin{array}{l:l}-1 \\
-12\end{array}$ & 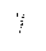 & 15 & 12 & $51 / 4$ & $?$ & 66 & 80 & -4 & 10 & 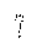 & 18 & 78 & -4 & 22 & ? & 独 & 3 \\
\hline$-4 \quad 10$ & 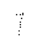 & 50 & 89 & $0 \%$ & 7 & 32 & 23 & -815 & 7 & 12 & 16 & -3 & 18 & 7 & 19 & 13 & -3 & 22 & "s & 16 & 12 \\
\hline-310 & 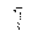 & 108 & 10 & 12 & 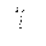 & 1 & ?! & -615 & 7 & $6 \mathrm{~g}$ & 80 & $-\cdots 2$ & 10 & 7 & $2 ?$ & 27 & $\cdots 2$ & 22 & 7 & $3 \hat{1}$ & 33 \\
\hline$-3 \quad 10$ & $\bar{\vdots}$ & a & $\%$ & 212 & 1 & 10 & 19 & -515 & $\uparrow$ & 25 & 26 & $\cdots$ & 10 & $?$ & 19 & 17 & $\cdots$ & 22 & $\bar{i}$ & 10 & 13 \\
\hline$\cdots$ & 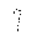 & 46 & 50 & 312 & $\because$ & 43 & 48 & $-4 \quad 15$ & $?$ & 3 & 75 & 0 & 18 & 7 & $70^{3}$ & 58 & $\hat{i}$ & $2 \%$ & 7 & tí & 40 \\
\hline i & $\because$ & $y$ & 33 & 18 & '! & 38 & 96 & -215 & "i & 45 & 48 & & 10 & 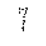 & 50 & 53 & 1 & 28 & ? & 14 & 10 \\
\hline 10 & 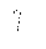 & 93 & $8 \%$ & 012 & 1 & 3 & 36 & $w 15$ & $\uparrow$ & 38 & 37 & 2 & 18 & $?$ & 27 & 30 & 2 & 22 & ? & 11 & 15 \\
\hline 30 & 7 & 34 & 30 & -413 & $\%$ & 33 & 36 & 015 & 7 & 28 & 29 & 3 & 18 & 7 & 11 & 2 & $-\sqrt{3}$ & 23 & $?$ & 19 & 21 \\
\hline 310 & 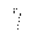 & 2 & 3 & 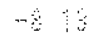 & 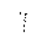 & १ै & 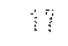 & 215 & 7 & 86 & 90 & 4 & 18 & i & 36 & 37 & -3 & 23 & $?$ & 20 & 20 \\
\hline 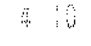 & $"$ & 29 & th & $-r i$ & $"$ & 48 & $4 \pi$ & 415 & 1 & 55 & 57 & 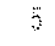 & 19 & "y & 3 & 35 & -2 & 23 & $?$ & 16 & 2 \\
\hline 510 & $\because$ & 83 & 9 & -6 & ? & : & Hi & 615 & 7 & 19 & 18 & 6 & 18 & i & $1 \%$ & 21 & $\cdots$ & 23 & 1 & 49 & स \\
\hline $\begin{array}{cc}-3 \\
-3\end{array}$ & $\because$ & 4 & 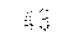 & -713 & 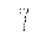 & 95 & 95 & -610 & "7 & 11 & 15 & -6 & 13 & 7 & 9 & 50 & 0 & 23 & 7 & 12 & 12 \\
\hline & 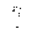 & 36 & 2 & $-\sqrt{4}$ & 1 & 80 & 80 & -516 & 7 & 28 & 28 & -4 & 19 & 7 & 39 & 30 & -10 & 0 & 8 & 39 & 37 \\
\hline$\ldots$ & 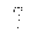 & 25 & $\because$ & -313 & $?$ & 62 & 63 & -416 & i & 60 & 67 & 0 & 10 & 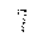 & 13 & $\mathrm{a}$ & -8 & 1 & i & 11 & $11 \hat{~}$ \\
\hline & 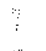 & 3 & 30 & -219 & 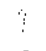 & 30 & 8 & -316 & 7 & 56 & 56 & 2 & 19 & $i$ & $\$ 4$ & 52 & -6 & 0 & 8 & 5.3 & $5 \hat{1}$ \\
\hline & $\vdots$ & 30 & 11 & $\cdots \vdots 13$ & 7 & $\%$ & 125 & -216 & ? & is & 19 & 3 & 13 & $?$ & $1 \mathfrak{1}$ & 10 & $\cdots \hat{\theta}$ & 0 & 8 & 04 & 83 \\
\hline & 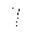 & 36 & 38 & 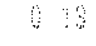 & $i$ & 12 & 12 & -16 & i & 45 & 64 & 4 & 19 & i & 30 & 31 & $\cdots$ & 0 & 6 & 偖学 & 18 \\
\hline-3 & 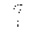 & 30 & 36 & 18 & 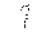 & 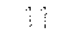 & 6 & 016 & 7 & $6 ?$ & 87 & 3 & 19 & 7 & 10 & 10 & 0 & 0 & , & 33 & 34 \\
\hline & 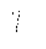 & 83 & 80 & 213 & 7 & 49 & 4 & 16 & 7 & 86 & 8 & -6 & 26 & ? & 19 & 15 & 2 & 0 & 8 & 30 & 6 \\
\hline ..: & $?$ & $\because$ & 7 & 313 & $?$ & 95 & 93 & 210 & 7 & 18 & 20 & -5 & 20 & $\dddot{r}$ & 20 & 26 & 6 & 0 & 8 & 56 & 54 \\
\hline 11 & 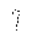 & 28 & 3 & $\because 4$ & 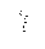 & 18 & 16 & 316 & 7 & $\hat{1} 1$ & 10 & -4 & 20 & 7 & 195 & î? & -11 & 1 & 8 & 19 & $2:$ \\
\hline 211 & 7 & 2 & 10 & 673 & i & 14 & 12 & 16 & 7 & 60 & 60 & $\cdots$ & 20 & $\overrightarrow{7}$ & 47 & 46 & -10 & 1 & 8 & 21 & 18 \\
\hline 311 & 7 & $8 \%$ & 86 & 30 & 7 & 11 & 5 & $5 \hat{0}$ & 7 & $1 / 4$ & 11 & $\ldots 2$ & 20 & $?$ & 17 & 12 & $\cdots$ & $\xi$ & k & 48 & \\
\hline
\end{tabular}

\begin{tabular}{|c|c|c|c|c|c|c|c|c|c|c|c|c|c|c|c|c|c|c|c|c|c|c|c|}
\hline$n$ & 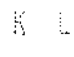 & $P$ & 8 & $\because$ & X & $\therefore$ & Po & $F$ & $\pi$ & is & i & 10 & FC & 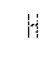 & is & i.. & 50 & 106 & 4 & 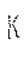 & & 0 & $\mathrm{PC}$ \\
\hline$\cdots$ & $\vdots$ & 3 & 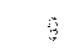 & -8 & 3 & 3 & 42 & 42 & -7 & 8 & 8 & 13 & $\delta$ & -3 & 7 & 8 & 10 & 8 & -1 & & 8 & $3 ?$ & 100 \\
\hline$-?$ & 1 & 89 & 67 & -1 & 3 & 8 & $2 \hat{1}$ & 22 & $-\hat{0}$ & 5 & 8 & 18 & 16 & -2 & $\bar{i}$ & 8 & 30 & 30 & 0 & 9 & 8 & 26 & 28 \\
\hline-6 & $\vdots$ & $\$ 2$ & 150 & -8 & 2 & 8 & 135 & 134 & -5 & 5 & $\ddot{d}$ & 106 & 120 & | & $?$ & 8 & 48 & 45 & 1 & 9 & 8 & 35 & 35 \\
\hline-5 & 1 & 95 & 8 & -3 & 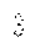 & 8 & 45 & 4 & $-\frac{14}{4}$ & $\pi$ & 8 & 43 & 43 & 0 & i & 8 & 20 & 30 & 2 & 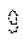 & 8 & 31 & 37 \\
\hline-4 & $\because$ & 5 & 10 & -4 & 3 & 3 & 85 & 85 & -3 & 5 & 8 & 19 & $4 B$ & 1 & \% & 8 & 17 & 13 & 3 & 9 & c & 100 & 103 \\
\hline$\cdots 3$ & 3 & 10 & 18 & -3 & 3 & 6 & 118 & Ih & -2 & 5 & 8 & 49 & 52 & 2 & $\overline{7}$ & 8 & 76 & 76 & 4 & 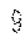 & 8 & 69 & 70 \\
\hline$-\gamma$ & 4 & औ) & 2 & -2 & 3 & 3 & 13 & 74 & -1 & 5 & 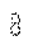 & 8 & 84 & 3 & 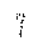 & 8 & 20 & $1 ?$ & 8 & 9 & 8 & 35 & 35 \\
\hline : & $1 c$ & $\hat{n}$ & $\theta \%$ & $-\because$ & $a$ & 0 & $\sin$ & १९s & $\mathrm{n}$ & $=$ & 0 & $\pi 0$ & $5:$ & $:$ & 7 & 0 & $\therefore$ & an & s & & $n$ & $\therefore$ & \\
\hline
\end{tabular}




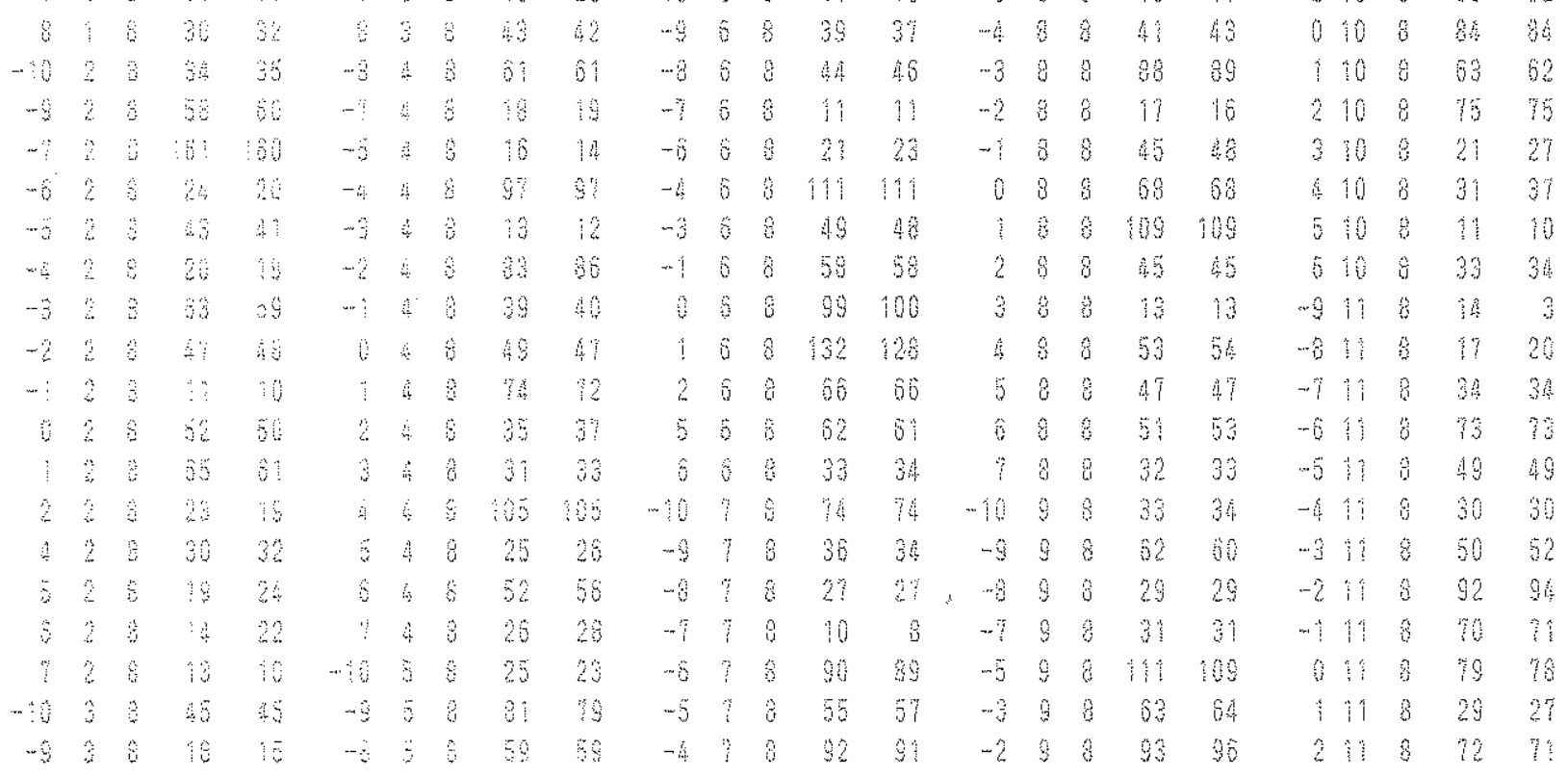

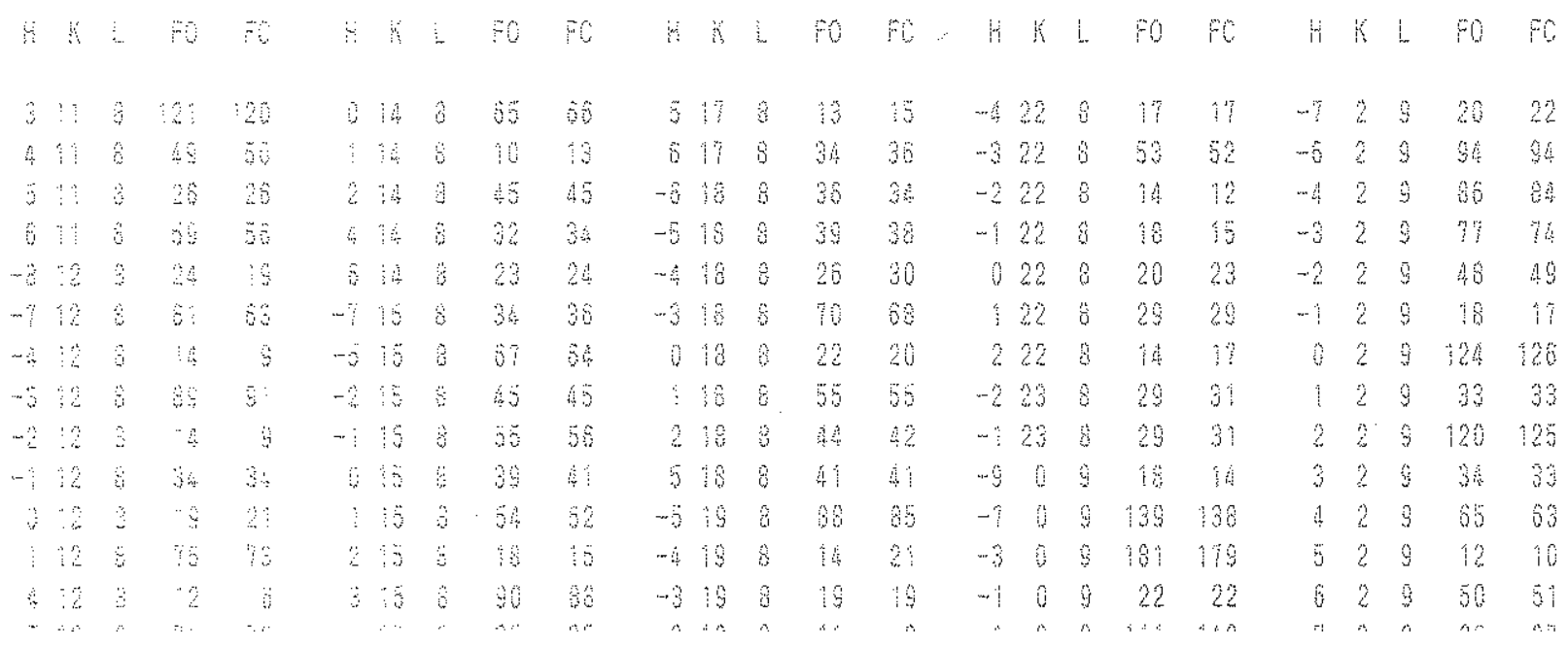




\begin{tabular}{|c|c|c|c|c|c|c|c|c|c|c|c|c|c|c|c|c|c|c|c|c|c|}
\hline$\cdots: 10$ & 3 & $10 !$ & 0 & $\cdots: 78$ & $\theta$ & 13 & $\beta$ & -320 & 8 & 38 & 30 & -4 & $i$ & 9 & 90 & 39 & -3 & 3 & 3 & 9 & 5 \\
\hline 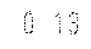 & 8 & 40 & $4 ?$ & 18 & $\because$ & 8 & $5 \%$ & $\cdots 220$ & 8 & 33 & 33 & $\cdots 3$ & 1 & 3 & 20 & 21 & $-?$ & 3 & 9 & 10 & 3 \\
\hline 48 & 3 & ? & 8 & : : : & 8 & 36 & 30 & 020 & 8 & 30 & 30 & -2 & 1 & 9 & 122 & 126 & $-i$ & 3 & 9 & 82 & 81 \\
\hline 213 & i. & 96 & 85 & 210 & 3 & 14 & 21 & 120 & 8 & $\hat{10}$ & 20 & -1 & $\hat{\imath}$ & 3 & 79 & 71 & 0 & 3 & 9 & 11 & 3 \\
\hline 13 & 8 & 9 & 3 & 510 & i & 38 & 37 & 220 & $\hat{B}$ & 48 & 48 & 0 & 1 & 9 & 37 & 34 & $\hat{\imath}$ & 3 & 3 & 23 & 20 \\
\hline मी & 8 & 80 & $8:$ & $-6 \quad 11$ & 8 & 45 & 4 & 420 & 8 & 28 & 2 & 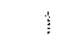 & 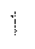 & 9 & $6:$ & 30 & $?$ & 3 & 9 & si & i! \\
\hline 513 & 3 & 30 & ज: & \%? & $\mathrm{a}$ & 29 & 32 & -621 & 8 & 20 & $3 \hat{1}$ & 2 & 1 & 9 & 32 & 70 & 3 & 3 & 9 & 74 & 71 \\
\hline 13 & 8 & 46 & 48 & -311 & 8 & 31 & 3 & \begin{tabular}{ll|l}
-4 & 21
\end{tabular} & 8 & 25 & 30 & 3 & $\hat{\imath}$ & 8 & Î & $\hat{1}$ & 4 & 3 & 3 & 47 & 89 \\
\hline-714 & 3 & 39 & 58 & -211 & 8 & 34 & 54 & -221 & $\hat{B}$ & in & 46 & 4 & 1 & 3 & 28 & 28 & 7 & 3 & 9 & 23 & 29 \\
\hline-8 & 8 & $\mathrm{~s}$ & a & 011 & $\mathrm{~s}$ & 48 & 50 & -21 & 8 & 12 & 18 & 8 & 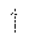 & s & 31 & 20 & -10 & is & 9 & 29 & 27 \\
\hline & 3 & 3 & 28 & $\vdots 17$ & $\partial$ & 9 & $\ddot{v}$ & 021 & 8 & 34 & 39 & 6 & 1 & 9 & 25 & 20 & -9 & 4 & 9 & 23 & 23 \\
\hline $\begin{array}{lll}4 & 14\end{array}$ & 8 & 3 & 18 & 211 & 8 & 30 & 38 & 121 & 8 & 18 & 20 & 1 & 1 & 9 & 25 & 28 & -8 & $i$ & $\hat{3}$ & 6 & 82 \\
\hline$-3 \quad \vdots 4$ & 8 & 8 & 12 & 311 & 8 & 30 & 86 & 221 & 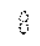 & $2 t$ & $2 A$ & -10 & 2 & 3 & $2 \hat{1}$ & 25 & $-r$ & 4 & 9 & 94 & \\
\hline-213 & 8 & 58 & 56 & $4: 1$ & 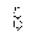 & 24 & 26 & 321 & 8 & 13 & 19 & -8 & 2 & 3 & & 103 & $-\overline{6}$ & 4 & 8 & $?$ & \\
\hline
\end{tabular}

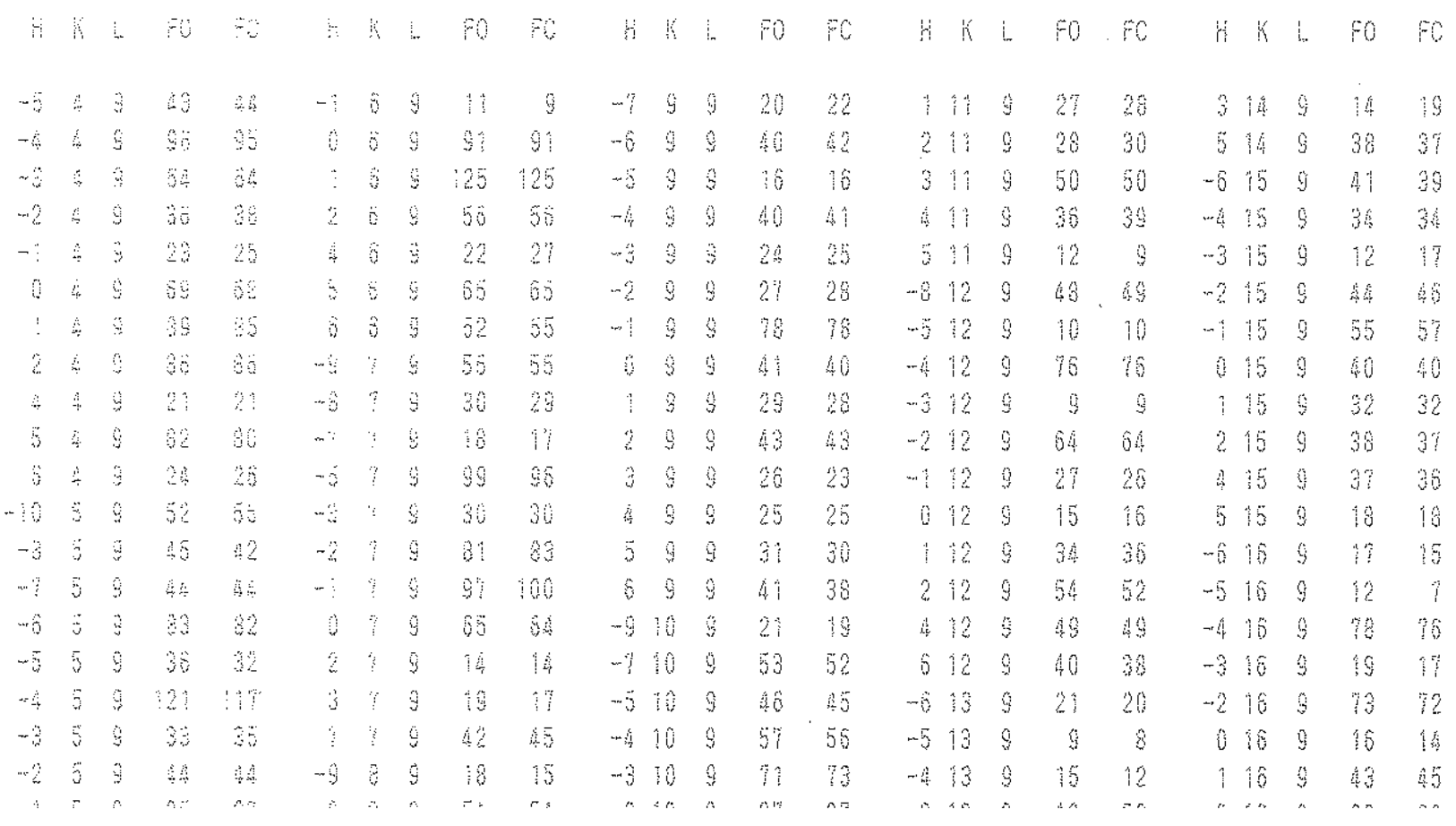




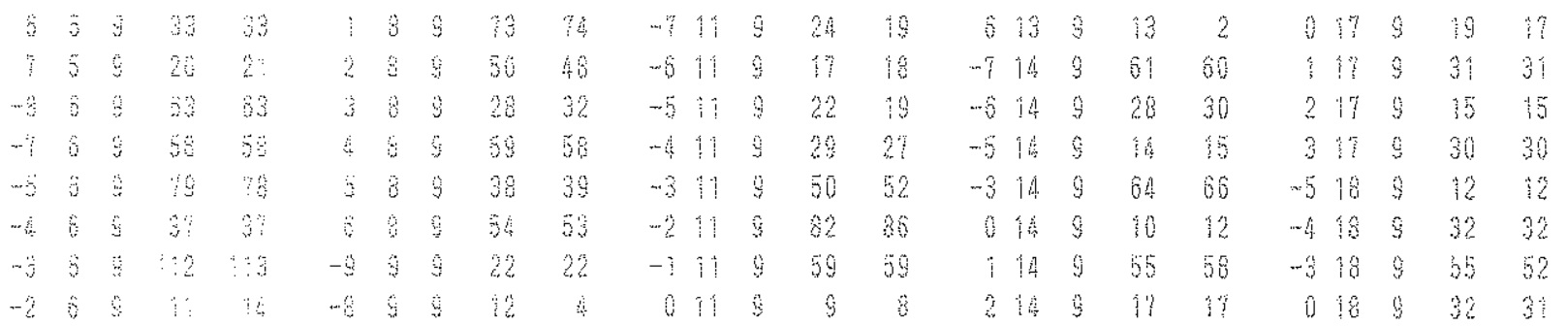

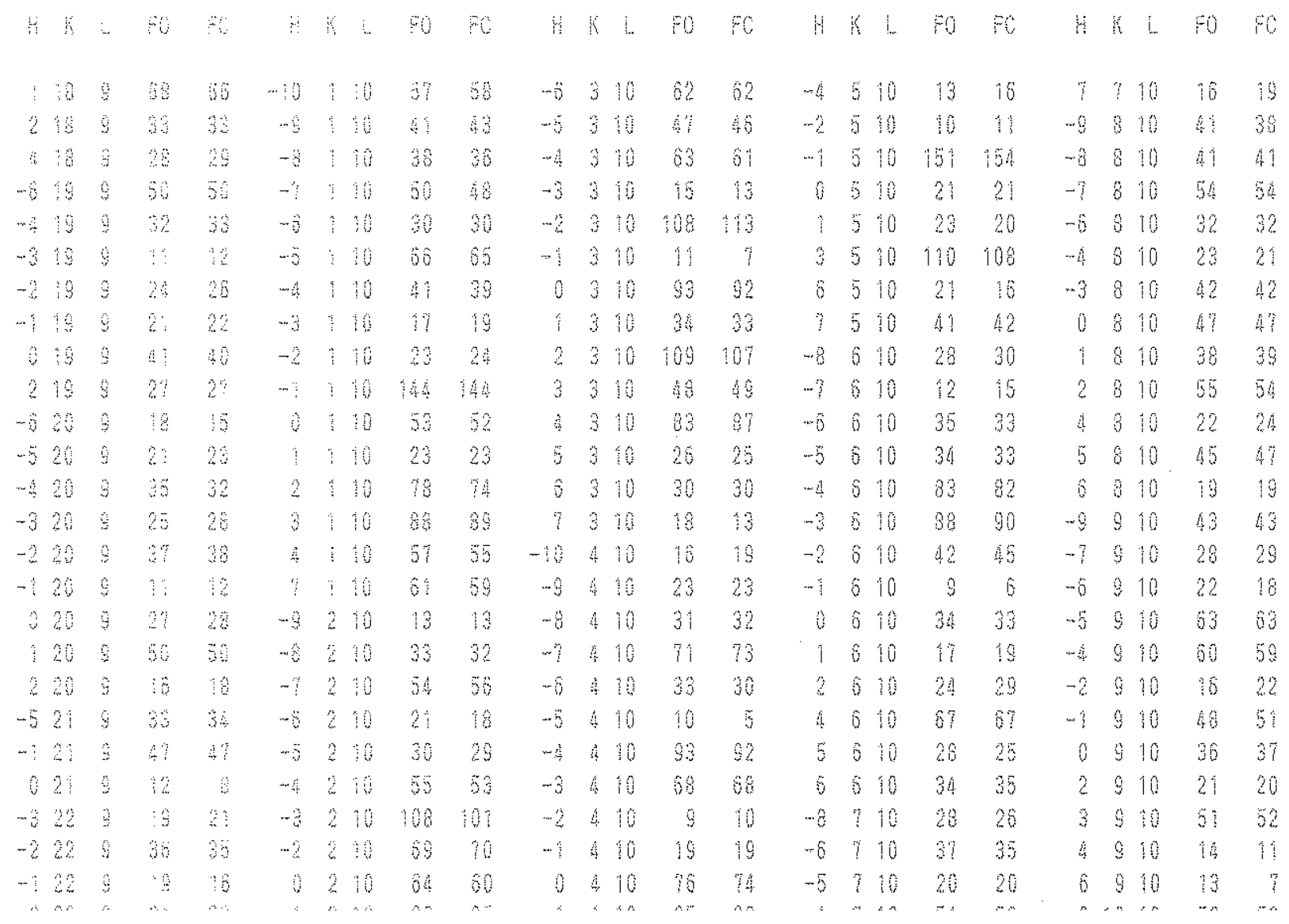




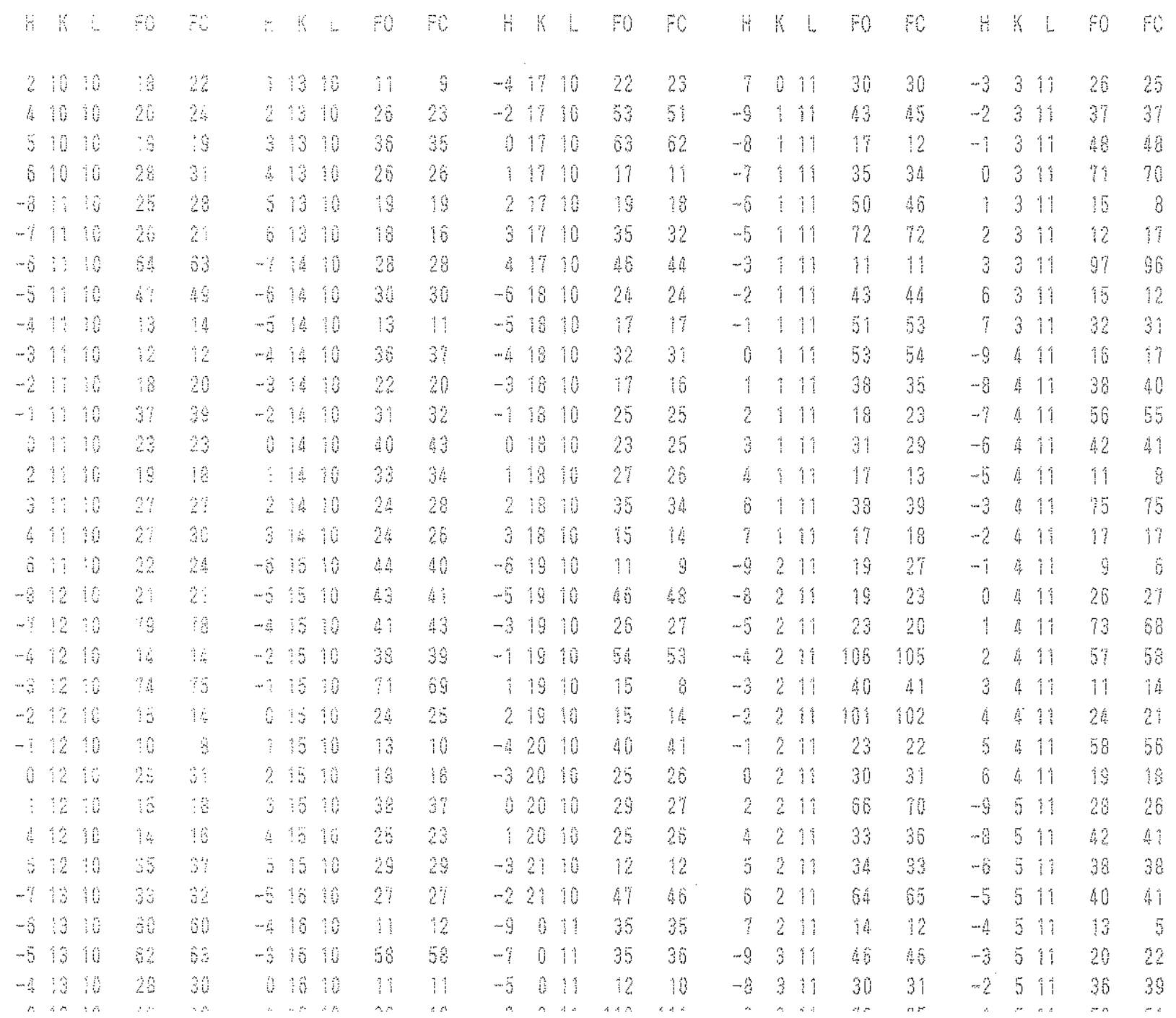




\begin{tabular}{|c|c|c|c|c|c|c|c|c|c|c|c|c|c|c|c|c|c|c|c|c|c|c|c|}
\hline$y$ & $\vdots$ & Fo & $\mathrm{PC}$ & $\mathrm{F}$ & $\mathrm{K}$ & 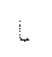 & $\mathrm{Fo}$ & 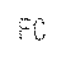 & $H$ & $\mathbb{R}$ & $\mathrm{L}$ & po & $F_{0}$ & $\mathrm{it}$ & $k$ & 1 & 60 & $p$ & $H$ & $K$ & $L$ & $j_{0}$ & $\mathrm{Fc}$ \\
\hline 2 & 311 & $A$ & $a n$ & -4 & 81 & & 25 & 26 & 5 & 10 & 11 & 45 & 4 & $-i$ & if & $1 \hat{1}$ & 32 & 35 & $-\overline{3}$ & 19 & 11 & 12 & 14 \\
\hline 3 & 5 & 14 & $\%$ & -3 & 81 & 1 & 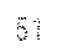 & 54 & 6 & 10 & 11 & 10 & $\hat{1}$ & 0 & 14 & $1 \hat{1}$ & 13 & 13 & $-\frac{1}{4}$ & 10 & 11 & 18 & $1 n$ \\
\hline 4 & $5: 1$ & 89 & 59 & $-z$ & $\mathrm{~B}$ & 11 & $5 \hat{\imath}$ & 63 & -7 & $\|$ & 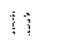 & $1 ?$ & 1 & 1 & 14 & $\hat{\imath}$ & 79 & 80 & -2 & 19 & $\|$ & 31 & 36 \\
\hline 5 & 511 & $\%$ & 10 & $\cdots$ & 8 & $\vdots$ & 12 & 11 & $\cdots \hat{0}$ & $\| \hat{~}$ & $1 \mathrm{i}$ & 60 & 60 & 2 & if & $1 \hat{1}$ & 15 & 20 & $\cdots 1$ & 19 & $\hat{1} 1$ & 130 & $1 / 4$ \\
\hline 3 & 31 & 30 & 8 & 0 & 8 & 1 & 77 & 76 & -3 & 1 & 11 & 77 & 78 & 3 & 1 & 1 & 18 & 10 & 0 & 19 & 11 & 25 & 28 \\
\hline-9 & 6 & 3 & 86 & 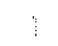 & 8 & (1) & 30 & 38 & -4 & 11 & $1 !$ & 53 & 83 & $-\frac{6}{0}$ & 15 & 1 & 51 & 48 & -2 & 20 & 1 & 25 & 23 \\
\hline-13 & $\ddot{\vec{u}}: 1$ & 36 & 37 & 2 & 31 & 11 & 58 & 59 & -3 & $\| \hat{1}$ & 1 & 10 & 21 & --5 & 15 & $\hat{i}$ & 30 & 23 & $\cdots$ & 0 & 12 & 80 & 64 \\
\hline (i & $5 \%$ & A & 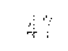 & 3 & 8 & 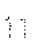 & 14 & 21 & $\sim$ & 11 & 11 & $4 ?$ & 47 & -4 & १ै & 11 & 35 & $3 \mathrm{H}$ & -6 & 0 & 12 & 35 & $\%$ \\
\hline-4 & 619 & १९ & 10 & $\vdots$ & 81 & 11 & 4 & 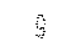 & 0 & $\uparrow$ & 1 & 42 & 33 & -2 & 15 & 11 & 38 & 41 & -2 & 0 & 12 & 18 & ; \\
\hline-3 & 611 & 56 & 60 & 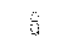 & 81 & $1 !$ & 38 & 38 & 2 & $1 \hat{1}$ & 1 & 13 & 13 & -1 & 15 & 1َ' 1َ| & 24 & 24 & 0 & $\hat{0}$ & 12 & 69 & 0 \\
\hline-2 & 51 & 30 & 183 & -8 & 3 & 1 & $B$ & 50 & 3 & 11 & 11 & 43 & an & 0 & 15 & $1 \hat{1}$ & 26 & 28 & 2 & 0 & 12 & 20 & 19 \\
\hline- & 51 & 8 & 3 & $\ldots$ & 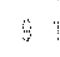 & $\because$ & 3 & 3 & 4 & 11 & 11 & 30 & 30 & $\dot{2}$ & 15 & $\hat{\imath} \hat{1}$ & 34 & 35 & 4 & 0 & 12 & 46 & dq \\
\hline$\vdots$ & a & 82 & 33 & -6 & 3 & 11 & 35 & 35 & $-\eta$ & 12 & 11 & 28 & 27 & 3 & 15 & 11 & 11 & 18 & 8 & 0 & 12 & 7 & 88 \\
\hline 3 & $6:$ & $\because$ & 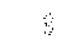 & 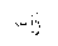 & 9 & 1 & 38 & 39 & $\cdots$ & 12 & 1 & 95 & 65 & 4 & 15 & 11 & 26 & $2 ?$ & -9 & $\hat{\imath}$ & 12 & 36 & 6 \\
\hline 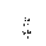 & $6:$ & ! & 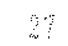 & $\cdots$ & 9 & 11 & 33 & 24 & -3 & 12 & 1 & 19 & 18 & -6 & 10 & $\hat{\imath}$ & 21 & 20 & $-\hat{3}$ & 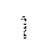 & 12 & 7 & $\eta$ \\
\hline 5 & 61 & औ & is & $\cdots$ & $\$$ & : & 34 & 36 & -4 & 12 & 1 & an & 46 & -4 & 16 & $\|$ & 28 & 24 & $-\cdots$ & 1 & 12 & 21 & 20 \\
\hline 8 & $\ddot{z}:$ & 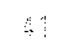 & $\$ 0$ & $\cdots$ & 9 & $\hat{\imath}$ & 48 & 5 & $\ldots$ & 12 & I" & 43 & 42 & $\ldots 3$ & 16 & 11 & 59 & $6 \hat{1}$ & $-\hat{0}$ & 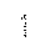 & 12 & 56 & 54 \\
\hline 't & 6 & : & 9 & $\cdots$ & 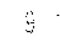 & $\because$ & 3 & 36 & -1 & 12 & $1 \hat{\imath}$ & $1 \%$ & 10 & $\cdots$ & 16 & 11 & 18 & 18 & $-\ddot{3}$ & $\hat{1}$ & 12 & 62 & \\
\hline$\cdots$ & Y : & $\because \%$ & $\%$ & 1 & 9 & 11 & 28 & 29 & 0 & 12 & 1 & 80 & 60 & 0 & 16 & $3 !$ & 5 & 10 & $-i$ & $\hat{1}$ & $\hat{1} 2$ & 50 & 49 \\
\hline-6 & 71 & 83 & 3 & $\vdots$ & 8 & 1 & 12 & 15 & ?. & 12 & 1 & 92 & 88 & 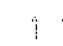 & 16 & 1 & 13 & 11 & -3 & $\hat{\imath}$ & 12 & 11 & 13 \\
\hline$\cdots$ & 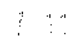 & $y$ & 4 & 2 & 9 & $1 \hat{!}$ & 20 & 39 & 3 & 12 & 1 & 13 & 4 & 2 & 10 & 11 & $2 !$ & 28 & $-\overline{2}$ & $\hat{\imath}$ & 2 & $3 \hat{3}$ & 60 \\
\hline-3 & 41 & 18 & $1 \%$ & 3 & 9 & $\because$ & 10 & $\|$ & : & 12 & 11 & 10 & 20 & -6 & 1 & 1 & 13 & 10 & $\cdots$ & 1 & 12 & 50 & 8 \\
\hline$\sim 3$ & ": :" & $: 6$ & $\because$ & 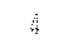 & 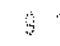 & $\because$ & 31 & $3 \%$ & -8 & 13 & $\|$ & 20 & 30 & -5 & 13 & 1 & 61 & 39 & i) & 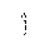 & 12 & 52 & 52 \\
\hline-3 & $" \hat{1}$ & $7 \%$ & 13 & $\ddot{z}$ & 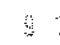 & 1 & 10 & 13 & -3 & 13 & 11 & $8 \%$ & 66 & -3 & $\Uparrow$ & 11 & 12 & $\hat{H}$ & $\hat{\mathrm{I}}$ & 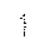 & 12 & 29 & 23 \\
\hline 3 & ? & : & $:$ & -3 & 10 & 1 & 33 & 30 & -4 & 13 & 11 & 45 & 47 & $-\bar{i}$ & 17 & 11 & 45 & 50 & $\stackrel{4}{4}$ & $i$ & 12 & 34 & 33 \\
\hline 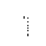 & 111 & 20 & 20 & $\cdots$ & $10^{\circ}$ & 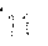 & 79 & 80 & -2 & 13 & 11 & 88 & SOC & 2 & $\eta$ & 11 & 琣 & 8 & 5 & $\hat{\mathrm{i}}$ & 12 & 32 & 27 \\
\hline 2 & : & :? & 3 & $-\ddot{0}$ & 10 & $\|$ & 2 & 23 & $\cdots$ & 13 & $1 \hat{1}$ & 27 & 29 & 3 & $1\}$ & 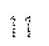 & $2 \sqrt{5}$ & 20 & -9 & 2 & 12 & औ & 17 \\
\hline 3 & 111 & 86 & 80 & -3 & 10 & 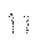 & 18 & $1 i$ & 0 & 13 & 1 & $\hat{1} \hat{\imath}$ & 20 & -6 & 10 & 11 & 28 & 27 & -8 & 2 & 12 & 3 & 35 \\
\hline 4 & Y: & $\because$ & 20 & -4 & 10 & 11 & 91 & 31 & 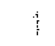 & 13 & 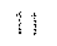 & 10 & 10 & -4 & 13 & $\hat{1} \hat{!}$ & 35 & $3 \hat{0}$ & $-i$ & 2 & 12 & 54 & 53 \\
\hline 5 & 11 & 3 & 86 & -8 & 10 & $1 \hat{\imath}$ & 36 & 9 & 2 & 13 & $\hat{1}$ & 22 & $2 \%$ & -3 & 10 & $\hat{\imath}$ & 22 & $2 \hat{1}$ & $-\mathrm{f}$ & 2 & $1 \%$ & 11 & $\hat{1}$ \\
\hline-3 & 8 & 38 & 10 & -2 & 10 & $\hat{\imath} \hat{\imath}$ & 71 & 16 & 3 & 13 & 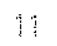 & 30 & 30 & -2 & 18 & 1 & 32 & 33 & -4 & 2 & 12 & 25 & 21 \\
\hline$\cdots ?$ & 811 & $b$ & $8 \%$ & 0 & 10 & $\hat{i} i$ & 35 & 36 & 4 & 13 & 11 & 25 & 23 & 0 & 13 & 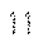 & 21 & 22 & -3 & 2 & 12 & 10 & \\
\hline-0 & 81 & 38 & 81 & $\vdots$ & 10 & 11 & 87 & 38 & -9 & 14 & 11 & 31 & 33 & 1 & 18 & 11 & 35 & 30 & -2 & 2 & 12 & 40 & \\
\hline$-\ddot{3}$ & $8 \%$ & 86 & 64 & $\hat{3}$ & 10 & १ו & 36 & 37 & $-\hat{j}$ & 14 & $1 \hat{1}$ & 49 & 50 & 2 & 10 & $\hat{11}$ & 18 & 16 & -1 & $?$ & 1 & 20 & \\
\hline
\end{tabular}




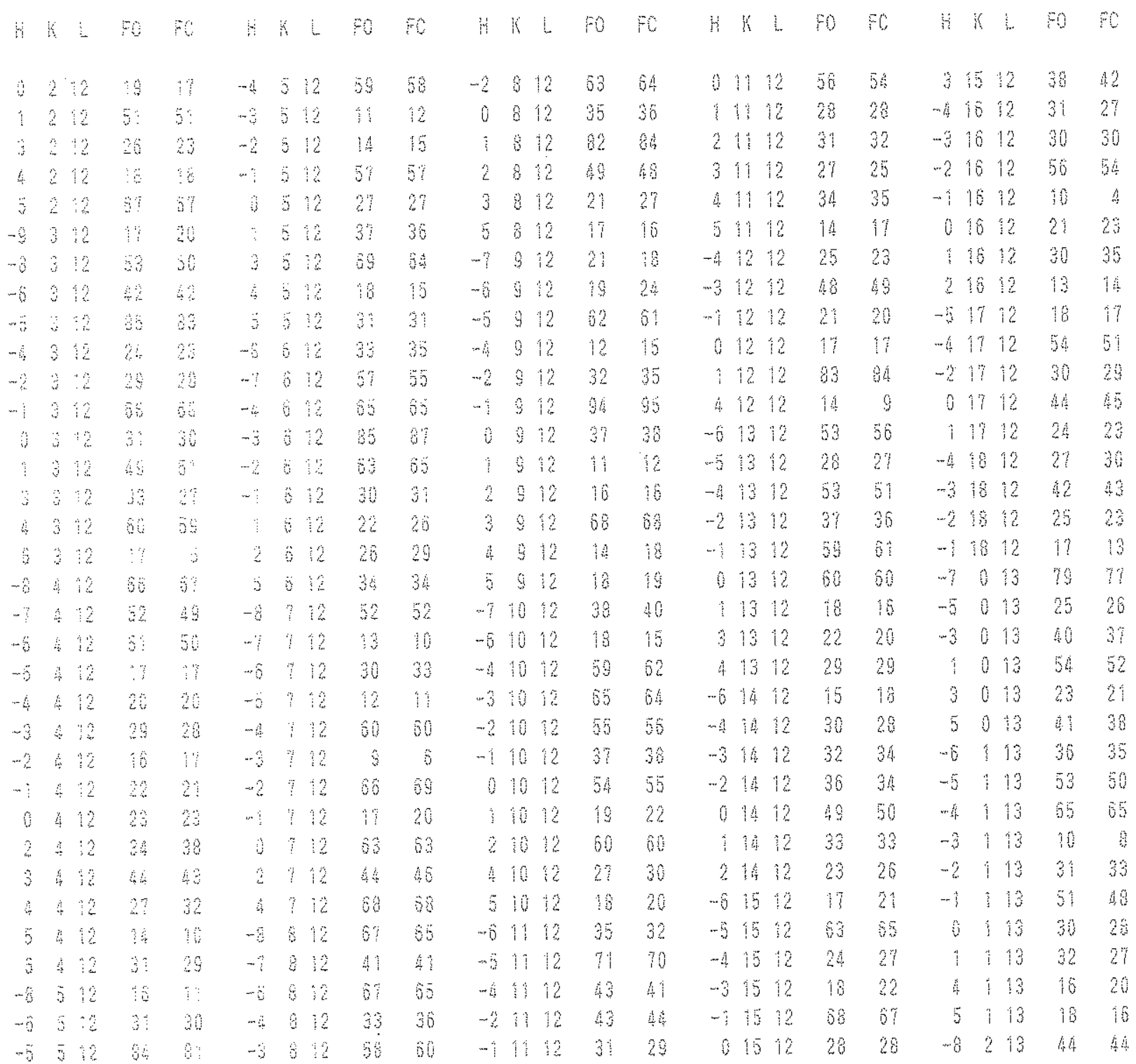




\begin{tabular}{|c|c|c|c|c|c|c|c|c|c|c|c|c|c|c|c|c|c|c|c|c|c|c|}
\hline 4 & $6:$ & 0 & 6 & $n$ & 8 & 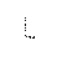 & $\mathrm{FO}$ & $\mathrm{PO}$ & 1 & $x$ & $L$ & Fo & 0 & $\hat{b i}$ & & & 50 & $p$ & H & & $L$ & 1 \\
\hline$\cdots "$ & 20 & 18 & ¿! & 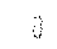 & $\ddot{3}$ & & 30 & 31 & $\theta$ & 3 & $\hat{1}$ & 28 & 29 & -3 & 16 & 13 & 51 & 51 & 1 & & 14 & 66 \\
\hline-6 & $\therefore 13$ & \%: & 16 & 2 & 8 & 13 & 53 & 81 & 2 & 9 & 13 & 39 & 38 & -1 & 14 & 13 & 20 & 26 & 3 & 2 & 14 & 6 \\
\hline$-\hat{\xi}$ & $\therefore \quad:$ & 3 & 18 & 3 & 5 & 13 & 11 & 15 & $\hat{i}$ & 9 & 13 & 38 & 35 & 0 & is & 13 & 13 & 10 & -6 & 3 & 14 & 23 \\
\hline$\cdots$ & 213 & 03 & 63 & $\div$ & $\ddot{z}$ & 13 & 48 & 15 & -6 & 10 & 13 & 13 & $\ddot{3}$ & 1 & i⿱ & 33 & 30 & 36 & -5 & 3 & 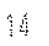 & 8 \\
\hline-3 & $\begin{array}{ll}30 \\
3\end{array}$ & 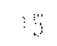 & $\%$ & -1 & 8 & 13 & 43 & 49 & -5 & 10 & 13 & In & 12 & -6 & 13 & 13 & 14 & 13 & $-\pi$ & 3 & 14 & \\
\hline$\cdots$ & 23 & 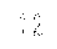 & 4 & -8 & 6 & 3 & 26 & 24 & -3 & 10 & 13 & 8 & 66 & -5 & 15 & 13 & 35 & 34 & -2 & 3 & $\hat{4}$ & 0 \\
\hline 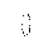 & 24 & 8 & 81 & $-\ddot{3}$ & 6 & n & 23 & 21 & -2 & 10 & 13 & 12 & $?$ & $\omega$ & 15 & 13 & 25 & 27 & -1 & 3 & 14 & $\overline{7}$ \\
\hline 3 & $2: 3$ & 88 & A: & -4 & $\ddot{\theta}$ & 13 & 26 & 26 & -1 & 10 & 13 & 31 & 32 & -3 & 15 & 13 & 15 & $\hat{A}$ & 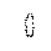 & 3 & 13 & 81 \\
\hline 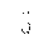 & $2: 0$ & 3 & & -3 & $\hat{3}$ & 19 & 12 & $\hat{1}$ & $i$ & 10 & 3 & 36 & 37 & -2 & 15 & 13 & 24 & 29 & $\hat{1}$ & 3 & 14 & i) \\
\hline-6 & 810 & 34 & 30 & -6 & $\ddot{b}$ & 13 & 42 & is & 2 & 10 & 13 & 37 & 30 & |1 & 15 & 3 & 30 & 33 & 3 & 3 & $\hat{4}$ & 8 \\
\hline 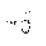 & $3: 3$ & 89 & 1 & $-!$ & 0 & 13 & 49 & 49 & 3 & 10 & 13 & 15 & 10 & 0 & 15 & 13 & 43 & 42 & 4 & 3 & $\hat{\imath}$ & 3 \\
\hline-4 & 313 & 8 & $\because$ & i & 6 & 3 & 48 & 48 & -3 & 11 & 13 & 33 & 36 & $\hat{i}$ & 15 & 13 & 13 & 20 & -7 & i & $\hat{\imath} \hat{a}$ & 50 \\
\hline-2 & $3 \%$ & -8 & $2:$ & 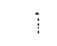 & 6 & 13 & 13 & 17 & $-\ddot{3}$ & 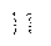 & 13 & & 30 & -2 & 10 & 13 & 43 & 43 & -6 & á & $\hat{n}$ & 10 \\
\hline$\cdots$ & 310 & 7 & 66 & 2 & $\hat{b}$ & 13 & 5 & 58 & $-\frac{1}{4}$ & 11 & $\hat{\uparrow}$ & 23 & 33 & -6 & 0 & 4 & 30 & 35 & -5 & 4 & 14 & 8 \\
\hline 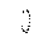 & 310 & $B$ & $4 ?$ & 3 & $b$ & 13 & 18 & 17 & -3 & 11 & 13 & 38 & 34 & $-x$ & 0 & 被 & 90 & 35 & $\cdots$ & $A$ & 4 & 32 \\
\hline 3 & 313 & 38 & 35 & -6 & 7 & 13 & 20 & 10 & $\cdots \hat{\mid}$ & $\hat{1} \hat{i}$ & $\hat{3}$ & 24 & 20 & -2 & 0 & $\hat{i}$ & 100 & 101 & -3 & 4 & 14 & 38 \\
\hline$\Delta$ & 313 & 2 & 26 & -5 & 7 & 13 & 20 & 40 & 0 & 1 & 13 & 3 & 33 & 0 & 0 & 33 & 45 & AY & $\cdots$ & $\hat{4}$ & 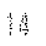 & 86 \\
\hline$\cdots$ & 613 & 38 & $3^{1}$ & ris & ' & 13 & $\hat{A}$ & 10 & 1 & 11 & 13 & 24 & 21 & 2 & $\theta$ & †育 & 48 & 65 & 0 & 4 & 14 & 13 \\
\hline$\leadsto \hat{\imath}$ & 40 & 87 & 56 & $\cdots$ & 7 & 13 & 32 & 12 & 2 & 11 & 13 & 18 & $\bar{\gamma}$ & 4 & () & 14 & 19 & 12 & 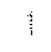 & it & in & 37 \\
\hline-6 & \& 19 & 30 & 33 & 3 & 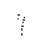 & 13 & 47 & 40 & $\hat{3}$ & 1 & 13 & 29 & 28 & -6 & 1 & 14 & 29 & 25 & 2 & 4 & 14 & 40 \\
\hline-3 & 40 & 2 & 2 & $\cdots$ & 8 & 3 & 23 & 21 & -6 & 12 & 13 & 47 & 44 & -5 & $i$ & 1 & 11 & 11 & -0 & 5 & $\hat{\imath}$ & s \\
\hline$-n$ & 413 & 53 & $5:$ & -6 & 0 & 13 & 28 & 28 & -5 & 12 & 13 & 19 & 18 & $-\frac{1}{4}$ & 1 & $\hat{1} \hat{\beta}$ & 32 & 31 & -5 & 5 & 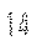 & 72 \\
\hline$\cdots$ & 413 & $4:$ & 4 & -4 & 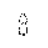 & 13 & 21 & 20 & $-\frac{4}{4}$ & 12 & 13 & 32 & 39 & -3 & 1 & 14 & 86 & 56 & -3 & 5 & 14 & $3 t_{7}$ \\
\hline-2 & 4 & 92 & 33 & -3 & 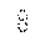 & 13 & 30 & 39 & -2 & 12 & 13 & 47 & 48 & -3 & 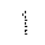 & j/ & 32 & 32 & -1 & 5 & $\hat{n}$ & 3 \\
\hline$\cdot$ & $\because 8$ & 05 & 52 & 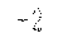 & 3 & 10 & 5 & 52 & 0 & 12 & 13 & 11 & 13 & -1 & 1 & 14 & 58 & 58 & 1 & 5 & 14 & 19 \\
\hline 2 & 13 & 80 & मह & 0 & 8 & 13 & 14 & 16 & 2 & 12 & 13 & 4 & 43 & 0 & 1 & 触 & 58 & 36 & 3 & $\overline{3}$ & 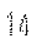 & 49 \\
\hline 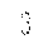 & 153 & 32 & 30 & $\vdots$ & 8 & 13 & 87 & 66 & $-b$ & 13 & 18 & 19 & 21 & 3 & 1 & 14 & 29 & $3 \hat{1}$ & -6 & 8 & 14 & 54 \\
\hline 5 & 413 & $3:$ & 32 & 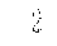 & 8 & 13 & 34 & 37 & $\cdots$ & 3 & 13 & 42 & $4 \hat{3}$ & 4 & $\hat{i}$ & 1 & 24 & 21 & $-i \hat{4}$ & 5 & 14 & 32 \\
\hline-1 & 613 & : & 8 & $\ddot{u}$ & 8 & 13 & 34 & 36 & $\cdots$ & 13 & 13 & 35 & 34 & .7 & 2 & 14 & 50 & 59 & -3 & 6 & 14 & 25 \\
\hline$-\hat{a}$ & $5 \uparrow$ & 20 & 36 & m & $\ddot{\vdots}$ & 13 & 21 & 22 & -2 & 13 & 13 & $\hat{\imath}$ & 16 & -6 & 2 & 14 & 24 & 22 & -2 & $\hat{0}$ & H & 33 \\
\hline-3 & 58 & 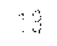 & 13 & -5 & 9 & 3 & 29 & 20 & -1 & 13 & 13 & 32 & $5 !$ & -3 & 2 & 14 & 64 & 85 & 0 & 6 & î & 15 \\
\hline-4 & 613 & को & 5 & -5 & 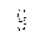 & 13 & $3 \%$ & 30 & 0 & 13 & 13 & 31 & 31 & 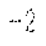 & 2 & $1 / 4$ & 32 & 28 & $\hat{\imath}$ & 6 & 4 & 20 \\
\hline-2 & 513 & 83 & 3 & -7 & 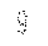 & $\vdots 3$ & $2 z$ & 22 & $\vdots$ & 13 & 13 & th & 15 & $\cdots$ & 2 & $\hat{1} i$ & 12 & is & 2 & 6 & 14 & 1 \\
\hline-1 & 518 & 9 & 30 & $\cdots:$ & 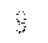 & 18 & 43 & 43 & $\cdots$ & 14 & 13 & 23 & 22 & $\mathrm{ij}$ & 2 & 14 & 17 & 21 & 3 & 6 & 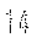 & 18 \\
\hline
\end{tabular}




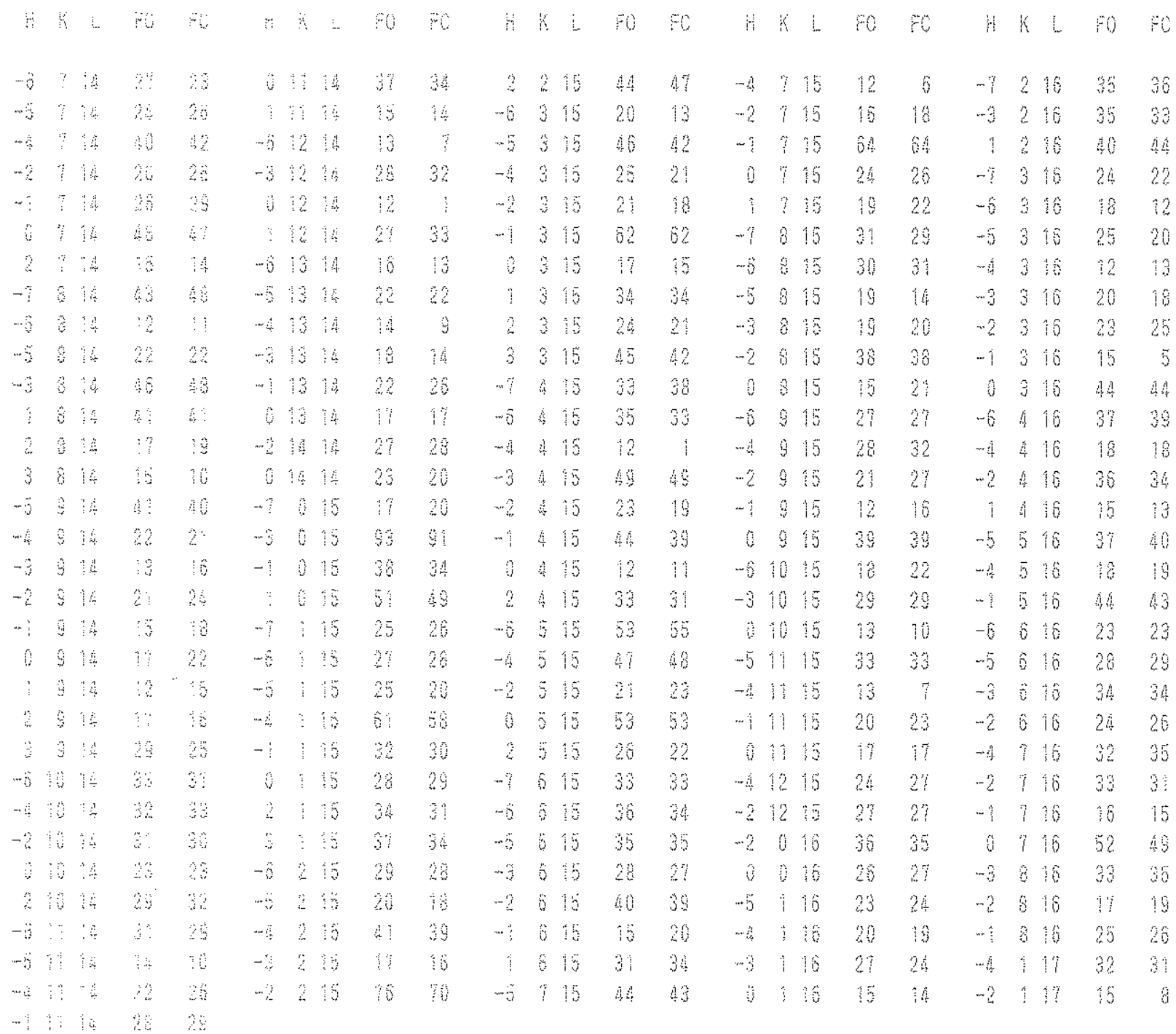

